\title{
Multiple Sign Changing Radially Symmetric Solutions in a General Class of Quasilinear Elliptic Equations *
}

\author{
Claudianor O. Alves ${ }^{\dagger}$ Jose V. A. Goncalves ${ }^{\ddagger}$ Kaye O. Silva ${ }^{\S}$
}

\begin{abstract}
In this paper we prove that the equation $-\left(r^{\alpha} \phi\left(\left|u^{\prime}(r)\right|\right) u^{\prime}(r)\right)^{\prime}=\lambda r^{\gamma} f(u(r)), 0<r<R$, where $\alpha, \gamma, \mathbf{R}$ are given real numbers, $\phi:(0, \infty) \rightarrow(0, \infty)$ is a suitable twice differentiable function, $\lambda>0$ is a real parameter and $f: \mathbf{R} \rightarrow \mathbf{R}$ is continuous, admits an infinite sequence of signchanging solutions satisfying $u^{\prime}(0)=u(R)=0$. The function $f$ is required to satisfy $t f(t)>0$ for $t \neq 0$. Our technique explores fixed point arguments applied to suitable integral equations and shooting arguments. Our main result extends earlier ones in the case $\phi$ is in the form $\phi(t)=|t|^{\beta}$ for an appropriate constant $\gamma$.
\end{abstract}

\section{Introduction}

We study the nonlinear eigenvalue problem

$$
\left\{\begin{aligned}
-\left(r^{\alpha} \phi\left(\left|u^{\prime}(r)\right|\right) u^{\prime}(r)\right)^{\prime} & =\lambda r^{\gamma} f(u(r)), \quad 0<r<R, \\
u^{\prime}(0) & =u(R)=0,
\end{aligned}\right.
$$

where $\lambda>0$ is a parameter, $f: \mathbb{R} \rightarrow \mathbb{R}$ is continuous and $\alpha, \gamma \in \mathbb{R}$ are suitable constants.

We shall assume that $\phi:(0, \infty) \rightarrow(0, \infty)$ is a twice differentiable, $C^{1}$-function, satisfying

$\left(\phi_{1}\right) \quad(\mathrm{i}) \quad t \phi(t) \rightarrow 0$ as $t \rightarrow 0$,

(ii) $t \phi(t) \rightarrow \infty$ as $t \rightarrow \infty$,

$\left(\phi_{2}\right) \quad t \phi(t)$ is strictly increasing in $(0, \infty)$,

$\left(\phi_{3}\right)$ there are constants $\gamma_{1}, \gamma_{2}>1$ such that

$$
\gamma_{1}-1 \leq \frac{(t \phi(t))^{\prime}}{\phi(t)} \leq \gamma_{2}-1, t>0
$$

Concerning $f$, the following conditions will be imposed:

$\left(f_{1}\right) t f(t)>0, t \neq 0$

\footnotetext{
* Partially supported by PROCAD/CAPES/UFG/UnB-Brazil

${ }^{\dagger}$ Claudianor O. Alves was supported by CNPQ/Brasil.

${ }^{\ddagger}$ Jose V. A. Goncalves was supported by CNPq/Brazil

${ }^{\S}$ Kaye O. Silva was supported by CAPES/Brazil
} 
$\left(f_{2}\right)$ there exists $d_{\infty}>0$ such that $f$ is nondecreasing in $\left(-\infty, d_{\infty}\right]$,

$$
\liminf _{\nu \rightarrow 0^{ \pm}} \int_{0}^{\nu}|f(t)|^{\frac{-1}{\gamma_{1}-1}} \operatorname{sgn}(f(t)) d t<\infty .
$$

Remark 1.1. We observe that condition $\left(f_{3}\right)$ is equivalent to the following:

$$
\max \left\{\int_{-x}^{0}[-f(t)]^{\frac{-1}{\gamma_{1}-1}} d t, \quad \int_{0}^{y}[f(t)]^{\frac{-1}{\gamma_{1}-1}} d t\right\}<\infty
$$

for each $x, y>0$, where $\gamma_{1}^{\prime}=\gamma_{1} /\left(\gamma_{1}-1\right)$.

Our main objective in this work is to prove the following result:

Theorem 1.1. Let $f \in C(\mathbb{R}) \cap C^{1}(\mathbb{R} \backslash\{0\})$. Assume $\left(\phi_{1}\right)$ - $\left(\phi_{3}\right),\left(f_{1}\right)-\left(f_{2}\right)$ and

$$
\gamma \geq \max \left\{\alpha, \frac{-\alpha}{\gamma_{1}-1}\right\} \text {. }
$$

Then there is a positive number $\Lambda$ such that for each $\lambda \in(0, \Lambda]$, problem $\left(P_{\lambda}\right)$ admits a positive solution $u_{0}$ and an infinite sequence $\left\{u_{\ell}\right\}_{\ell=1}^{\infty}$ of solutions satisfying:

$$
u_{l}(0)=d_{\ell},
$$

$u_{\ell}$ has precisely $\ell$ zeroes in $(0, R)$,

where $\left\{d_{\ell}\right\}_{\ell=1}^{\infty}$ is an infinite sequence of real numbers such that

$$
d_{\infty}>d_{1}>\cdots>d_{\ell}>\cdots>0
$$

The proof of Theorem 1.1 is strongly based on the shooting method. In this regard, consider the initial value problem

$$
\left\{\begin{array}{l}
-\left(r^{\alpha} \phi\left(\left|u^{\prime}(r)\right|\right) u^{\prime}(r)\right)^{\prime}=\lambda r^{\gamma} f(u(r)), \quad r>0, \\
u(0)=d, \quad u^{\prime}(0)=0,
\end{array}\right.
$$

where $d \in\left(0, d_{\infty}\right]$.

The auxiliary result below will play a crucial role in this work.

Theorem 1.2. Let $f \in C(\mathbb{R}) \cap C^{1}(\mathbb{R} \backslash\{0\})$. Assume $\left(\phi_{1}\right)-\left(\phi_{3}\right),(\gamma, \alpha)$ and $\left(f_{1}\right)-\left(f_{2}\right)$. Then there exists a positive number $\Lambda=\Lambda\left(d_{\infty}\right)$ such that for each $\lambda \in(0, \Lambda]$, problem $\left(P_{\lambda, d}\right)$ has a unique solituon $u(\cdot, d, \lambda)=u(\cdot, d) \in C^{1}([0, \infty))$. In addition, for each $d \in\left(0, d_{\infty}\right]$, there is a sequence $\left\{z_{\ell}\right\}_{\ell=1}^{\infty}$ of zeroes of $u(\cdot, d), z_{\ell}=z_{\ell}(d)$, such that

$$
\begin{aligned}
& z_{1}\left(d_{\infty}\right) \geq R, \quad u(r, d)>0 \text { if } 0<r<z_{1}(d), \\
& z_{1}(d)<z_{2}(d)<\cdots<z_{\ell}(d)<\cdots, \\
& u^{\prime}(r, d)<0 \text { if } 0<r \leq z_{1}(d), u(r, d) \neq 0 \text { if } z_{\ell}<r<z_{\ell+1} \text { and } u^{\prime}\left(z_{\ell}, d\right) \neq 0, \\
& z_{\ell}(d) \rightarrow 0 \text { as } d \rightarrow 0 \text { and } z_{\ell}(d) \rightarrow z_{\ell}(\underline{d}) \text { as } d \rightarrow \underline{d}, \underline{d} \in\left(0, d_{\infty}\right],
\end{aligned}
$$

if $\underline{d} \in\left(0, d_{\infty}\right]$ and $u(\cdot, \underline{d})$ has $k$ zeroes in $(0, R)$ then $u(\cdot, d)$ has at most $k+1$ zeroes in $(0, R)$ whenever $d<\underline{d}, d$ close enough to $\underline{d}$. 


\section{Background}

Consider the problem

$$
\left\{\begin{aligned}
-\operatorname{div}\left(a(x)|\nabla u(x)|^{\beta} \nabla u(x)\right) & =\lambda b(x) f(u), \quad x \in B_{R}, \\
u(x) & =0, \quad x \in \partial B_{R},
\end{aligned}\right.
$$

where $B_{R} \subset \mathbf{R}^{\mathbf{N}}$ is the ball of radius $R$ centered at the origin, the functions $a, b$ are radially symmetric and $\beta>-1$. Making $a=b \equiv 1, \beta=p-2$ with $1<p<\infty$ and $\lambda=1$, $P 1_{\lambda}$ becomes

$$
\left\{\begin{array}{c}
-\left(r^{N-1}\left|u^{\prime}(r)\right|^{p-2} u^{\prime}(r)\right)^{\prime}=r^{N-1} f(u(r)), \quad 0<r<R, \\
u^{\prime}(0)=u(R)=0 .
\end{array}\right.
$$

It was shown in [6] that if $f(t)=|t|^{\delta-1} t$ with $1<\delta+1<p<N$ then (P2) has infinitely many nodal solutions.

In [3], it was shown that the more general problem

$$
\left\{\begin{array}{l}
-\left(r^{\alpha}\left|u^{\prime}(r)\right|^{\beta} u^{\prime}(r)\right)^{\prime}=\lambda r^{\gamma} f(u(r)), \quad 0<r<R \\
u^{\prime}(0)=u(R)=0
\end{array}\right.
$$

admits infinitely many solutions if $\lambda$ is positive and small enough,

$$
\beta>-1, \quad \gamma \geq \max \left\{\alpha, \frac{-\alpha}{\beta+1}\right\},
$$

and conditions $\left(f_{1}\right),\left(f_{2}\right)$ and a stronger form of $\left(f_{3}\right)$ hold.

Regarding ( $(\underline{P 3})$, an example of a function safistying $\left(f_{1}\right),\left(f_{2}\right),\left(f_{3}\right)$ with $\beta>0$ is $f(t)=\operatorname{arctg}(t)$.

As was pointed out by Clement, Figueiredo \& Mitidieri [8] the operator

$$
\left(r^{\alpha}\left|u^{\prime}(r)\right|^{\beta} u^{\prime}(r)\right)^{\prime}
$$

represents the radial form of the well known operators:

$p$-Laplacian with $1<p<N$ when $\alpha=N-1, \beta=p-2$,

$k$-Hessian with $1 \leq k \leq N$ when $\alpha=N-k, \beta=k-1$.

The problem

$$
\left\{\begin{aligned}
-\Delta_{\Phi} u & =\lambda f(u) \text { in } B \\
u & =0 \text { on } \partial B
\end{aligned}\right.
$$

where

$$
\Phi(t)=\int_{0}^{t} s \phi(s) d s
$$

$\Delta_{\Phi}$ is the $\Phi$-Laplacian operator namely

$$
\Delta_{\Phi} u=\operatorname{div}(\phi(|\nabla u|) \nabla u)
$$


and $B \subset R^{N}$ is the ball of radius $R$ centered at the origin, was addressed by many authors (see e.g. Fukagai \& Narukawa[4] and its references). A weak solution of $(\Phi)$ is by definition an element $u \in W_{0}^{1, \Phi}(B)$ (the usual Orlicz-Sobolev space) such that

$$
\int_{B} \phi(|\nabla u|) \nabla u \nabla v d x=\lambda \int_{B} f(u) v d x, v \in W_{0}^{1, \Phi}(B) .
$$

The radially symmetric form of $(\Phi)$ is

$$
\left\{\begin{array}{c}
-\left(r^{N-1} \phi\left(\left|u^{\prime}(r)\right|\right) u^{\prime}(r)\right)^{\prime}=\lambda r^{N-1} f(u(r), 0<r<R \\
u^{\prime}(0)=u(R)=0
\end{array}\right.
$$

which is a special case of $\left(P_{\lambda}\right)$, (see further remarks in the Appendix).

Theorem 1.1 extends the main results of [3], 6], in the sense that we were able to treat both with a more general class of quasilinear operators and a broader class of terms $f$.

Problems like $\left(P_{\lambda}\right)$ have been investigated by many authors and we would like to refer the reader to Saxton \& Wei [16], Castro \& Kurepa [7, Cheng [5], Strauss [14, Ni \& Serrin [12, Castro, Cóssio \& Neuberger [9, Fukagai \& Narukawa [4], Mihailescu \& Radulescu [10, 11] and their references. Here, we would point out that in [4, Fukagai \& Narukawa have mentioned that this type of problem appears in some fields of physics, such as, nonlinear elasticity, plasticity and generalized Newtonian fluids.

\section{Proof of Theorem 1.1}

Take $\lambda \in(0, \Lambda]$ where $\Lambda>0$ is given in Theorem 1.2. We proceed in two steps:

Step 1. (Existence of a positive solution of $\left(\overline{P_{\lambda}}\right)$.) Let $d \in\left(0, d_{\infty}\right]$. We shall use the notations in Theorem 1.2. So $z_{1}=z_{1}(d)$ denotes the first zero of $u(\cdot)=u(\cdot, d)$. Set

$$
A_{0}=\left\{d \in\left(0, d_{\infty}\right] \mid z_{1}(d) \geq R\right\} \text { and } d_{0}:=\inf A_{0} .
$$

By (1.4) in theorem $1.2, z_{1}\left(d_{\infty}\right) \geq R$. So $A_{0} \neq \emptyset$. We will show that

$$
d_{0}>0 \text { and } z_{1}\left(d_{0}\right)=R \text {. }
$$

Indeed, assume on the contrary that $d_{0}=0$. Take a sequence $\left(d_{j}\right) \subseteq A_{0}$ such that $d_{j} \rightarrow 0$. By (1.5), $z_{1}\left(d_{j}\right) \rightarrow 0$, which is a contradiction.

Now, assume $z_{1}\left(d_{0}\right)>R$. Pick a sequence $\left(d_{j}\right) \subseteq\left(0, d_{\infty}\right]$ such that $d_{j}<d_{0}$ and $d_{j} \rightarrow d_{0}$. Applying (1.5) we infer that $z_{1}\left(d_{j}\right) \rightarrow z_{1}\left(d_{0}\right)$. Once $z_{1}\left(d_{0}\right)>R$, it follows that $z_{1}\left(d_{j}\right)>R$, which shows that $d_{j} \in A_{0}$. But this contradicts the definition of $d_{0}$. Therefore $z_{1}\left(d_{0}\right)=R$ and this completes the proof of (3.1). As a byproduct there is a positive solution of $P_{\lambda}$.

Step 2. (Existence of an infinite sequence of sign-changing solutions of $\left(P_{\lambda}\right)$.) At first consider

$$
A_{1}:=\left\{d \in\left(0, d_{0}\right] \mid z_{1}(d)<R, z_{2}(d) \geq R\right\} \text { and } d_{1}:=\inf A_{1} .
$$

We claim that 


$$
\begin{array}{r}
A_{1} \neq \emptyset, \quad 0<d_{1}<d_{0}, \\
z_{1}\left(d_{1}\right)<R, \quad z_{2}\left(d_{1}\right)=R .
\end{array}
$$

Let us show at first that $A_{1} \neq \emptyset$. Indeed, by Step $1 z_{1}\left(d_{0}\right)=R$. By (1.6), if $d \in\left(0, d_{0}\right)$ with $d$ close to $d_{0}$ then $u(\cdot, d)$ has at most one zero in $(0, R)$. Assume by contradiction that $u(\cdot, d)$ has no zero in $(0, R)$. Then $z_{1}(d) \geq R$ with $d<d_{0}$, impossible. It follows that $u(\cdot, d)$ has precisely one zero in $(0, R)$ and so $d \in A_{1}$, showing that $A_{1} \neq \emptyset$.

To show that $d_{1}>0$, assume by contradiction that there is a sequence $\left\{d_{j}\right\} \subset A_{1}$ such that $d_{j} \rightarrow 0$. By (1.5),$z_{2}\left(d_{j}\right) \rightarrow 0$ contradicting $z_{2}\left(d_{j}\right) \geq R$.

It follows from $z_{1}\left(d_{0}\right)=R$ and definition of $A_{1}$ that $d_{1}<d_{0}$. Therefore $0<d_{1}<d_{0} \leq d_{\infty}$.

It remains to show that $z_{1}\left(d_{1}\right)<R$ and $z_{2}\left(d_{1}\right)=R$. To do it, let $\left\{d_{j}\right\} \subseteq A_{1}$ such that $d_{j} \rightarrow d_{1}$, so that $z_{1}\left(d_{j}\right) \rightarrow z_{1}\left(d_{1}\right) \leq R$ and $z_{2}\left(d_{j}\right) \rightarrow z_{2}\left(d_{1}\right) \geq R$.

If $z_{1}\left(d_{1}\right)=R$ then $u\left(\cdot, d_{1}\right)$ has no zeros in $(0, R)$. By (1.6), if $d<d_{1}$ and $d$ is close to $d_{1}, u(\cdot, d)$ has at most one zero in $(0, R)$. If $u(\cdot, d)$ has one zero then we have $d<d_{1}$ and $d \in A_{1}$, a contradiction. On the other hand, if $u(\cdot, d)$ has no zero then $d \geq d_{0}>d_{1}$ which is again a contradiction. Therefore, $z_{1}\left(d_{1}\right)<R$. Now, assume by contradiction that $z_{2}\left(d_{1}\right)>R$. Let $d_{j} \rightarrow d_{1}$ with $d_{j}<d_{1}$. Then, $z_{1}\left(d_{j}\right) \rightarrow z_{1}\left(d_{1}\right)<R$ and in addition, $z_{2}\left(d_{j}\right) \rightarrow z_{2}\left(d_{1}\right)>R$, so that, $z_{1}\left(d_{j}\right)<R$ and $z_{2}\left(d_{j}\right)>R$ for large $j$, which is impossible. Thus $z_{2}\left(d_{1}\right)=R$.

By induction, iterating the arguments above, we construct a sequence $\left\{d_{\ell}\right\}_{\ell=1}^{\infty} \subseteq\left(0, d_{\infty}\right]$ such that

$$
\begin{array}{r}
0<\cdots<d_{\ell}<\cdots<d_{1}<d_{\infty}, \\
z_{\ell}\left(d_{\ell}\right)<R, z_{\ell+1}\left(d_{\ell}\right)=R,
\end{array}
$$

with $d_{\ell}:=\inf A_{\ell}$, where

$$
A_{\ell}:=\left\{d \in\left(0, d_{\ell}\right] \mid z_{\ell}(d)<R, z_{\ell+1}(d) \geq R\right\}
$$

This ends the proof of step 2.

To finish the proof of Theorem 1.1. we use steps 1 and 2 to conclude that for $\lambda \in(0, \Lambda]$ the functions given by Theorem [1.2, namely $u_{\ell}(\cdot)=u\left(\cdot, d_{\ell}\right) \in C^{1}([0, R])$ for $\ell \geq 1$, satisfy

$$
\begin{gathered}
r^{\alpha} \phi\left(\left|u_{\ell}^{\prime}(r)\right|\right) u_{\ell}^{\prime}(r) \text { is differentiable, } \\
-\left(r^{\alpha} \phi\left(\left|u_{\ell}^{\prime}(r)\right|\right) u_{\ell}^{\prime}(r)\right)^{\prime}=\lambda r^{\gamma} f\left(u_{\ell}(r)\right), 0<r<R, \\
u_{\ell}^{\prime}(0)=0 \text { and } u_{\ell}(R)=0,
\end{gathered}
$$

that is, $u_{l}$ is a classical solution of $\left(P_{\lambda}\right], u_{\ell}$ has precisely $\ell$ zeroes in $(0, R)$ and so

$$
u_{0}, u_{1}, u_{2}, \cdots
$$

is an infinite sequence of solutions of $\left(P_{\lambda}\right)$ as claimed in the statement of Theorem 1.1 


\section{Proof of Theorem 1.2}

At first we set

$$
\Phi(t)=\int_{0}^{t} s \phi(s) d s, \quad H(t)=t \Phi^{\prime}(t)-\Phi(t), \quad F(t)=\int_{0}^{t} f(s) d s
$$

The results below will play a crucial role in this paper.

Lemma 4.1. Assume $(\overline{\gamma, \alpha})$ and let $d \in\left[0, d_{\infty}\right], \lambda>0$ and $T>0$. If $u$ is a solution of $\overline{P_{\lambda, d}}$ in $[0, T]$, then

$$
\begin{gathered}
H\left(\left|u^{\prime}(r)\right|\right) \leq \lambda r^{\gamma-\alpha}[F(d)-F(u(r))], r \geq 0 . \\
F(u(r)) \leq F(d) \text { for } r \in[0, T]
\end{gathered}
$$

Lemma 4.2. Assume that $\left(\phi_{1}\right)-\left(\phi_{3}\right)$, $(\overline{\gamma, \alpha})$ and $\left(f_{1}\right)-\left(f_{2}\right)$ holds. If $f \in C(\mathbb{R}) \cap C^{1}(\mathbb{R} \backslash\{0\})$ and $d \in\left(0, d_{\infty}\right]$, then problem $\overline{\left(P_{\lambda, d}\right)}$ has a unique solution $u(\cdot, d, \lambda)=u(\cdot, d)=u(\cdot) \in C^{1}([0, \infty))$. In addition,

$$
\begin{aligned}
& \text { if } d_{0} \in\left(0, d_{\infty}\right] \text { then } u(r, d) \rightarrow u\left(r, d_{0}\right) \text { as } d \rightarrow d_{0} \text {, uniformly in }[0, T] \text { for } T>0 \text {, } \\
& \text { if } d_{0} \in\left(0, d_{\infty}\right] \text { then } u^{\prime}(r, d) \rightarrow u^{\prime}\left(r, d_{0}\right) \text { as } d \rightarrow d_{0} \text {, uniformly on compact } \\
& \text { subsets of }(0, \infty],
\end{aligned}
$$

\subsection{Proofs of Lemmas 4.1 and 4.2}

Integrating the equation in $\left(\overline{P_{\lambda, d}}\right)$ we get to

$$
\phi\left(\left|u^{\prime}(r)\right|\right) u^{\prime}(r)=-r^{-\alpha} \int_{0}^{r} \lambda t^{\gamma} f(u(t)) d t, r>0 .
$$

Setting

$$
h(t):=t \phi(t),
$$

we see that $h$ is invertible with differentiable inverse $h^{-1}$. Then,

$$
\begin{gathered}
u^{\prime}(r)=h^{-1}\left(-r^{-\alpha} \int_{0}^{r} \lambda t^{\gamma} f(u(t)) d t\right) \text { if } u^{\prime}(r)>0, \\
u^{\prime}(r)=-h^{-1}\left(-r^{-\alpha} \int_{0}^{r} \lambda t^{\gamma} f(u(t)) d t\right) \quad \text { if } u^{\prime}(r)<0,
\end{gathered}
$$

Once $f$ is continuous and $\gamma \geq \alpha$, we conclude from the above equalities that $u \in C^{1}$.

Proof of Lemma 4.1. From (4.7) and (4.8), we infer that $u \in C^{2}$ at the points $r>0$ where $u^{\prime}(r) \neq 0$. Computing derivatives in $\left(\overline{P_{\lambda, d}}\right)$ and multiplying the resulting equality by $u^{\prime}(r)$, we are led to

$$
-\alpha r^{\alpha-1} \phi\left(\left|u^{\prime}(r)\right|\right)\left|u^{\prime}(r)\right|^{2}-r^{\alpha} \frac{d}{d t} h\left(\left|u^{\prime}(r)\right|\right) u^{\prime}(r) u^{\prime \prime}(r)=\lambda r^{\gamma} f(u(r)) u^{\prime}(r), u^{\prime}(r) \neq 0 .
$$


Consider the functional $E:[0, \infty) \rightarrow \mathbb{R}$ defined by

$$
E(0)=\lambda F(d) \text { and } E(r)=r^{\alpha-\gamma}\left[H\left(\left|u^{\prime}(r)\right|\right)\right]+\lambda F(u(r)), r>0,
$$

where $H(t)=t \Phi^{\prime}(t)-\Phi(t)=\int_{0}^{t} h^{\prime}(s) s d s$. Note that

$$
E^{\prime}(r)=r^{\alpha-\gamma}\left[H\left(\left|u^{\prime}(r)\right|\right)\right]^{\prime}+(\alpha-\gamma) r^{\alpha-\gamma-1} H\left(\left|u^{\prime}(r)\right|\right)+\lambda f(u(r)) u^{\prime}(r), r>0
$$

and

$$
\left[H\left(\left|u^{\prime}(t)\right|\right)\right]^{\prime}=\frac{d}{d t} h\left(\left|u^{\prime}(r)\right|\right) u^{\prime}(r) u^{\prime \prime}(r), u^{\prime}(r) \neq 0 .
$$

Therefore from (4.9),

$$
E^{\prime}(r)=(\alpha-\gamma) r^{\alpha-\gamma-1} H\left(\left|u^{\prime}(r)\right|\right)-\alpha r^{\alpha-\gamma-1} \phi\left(\left|u^{\prime}(r)\right|\right)\left|u^{\prime}(r)\right|^{2} u^{\prime}(r) \neq 0 .
$$

From Lemma 5.6 in the Appendix, the last inequality combined with hypothesis $(\overline{\gamma, \alpha})$ gives

$$
E^{\prime}(r) \leq \frac{\gamma_{1}-1}{\gamma_{1}}(\alpha-\gamma) r^{\alpha-\gamma-1} \phi\left(\left|u^{\prime}(r)\right|\right)\left|u^{\prime}(r)\right|^{2}-\alpha r^{\alpha-\gamma-1} \phi\left(\left|u^{\prime}(r)\right|\right)\left|u^{\prime}(r)\right|^{2}<0, u^{\prime}(r) \neq 0 .
$$

Next, we will prove that $E$ is continuous at the origin and therefore, as $E$ is non-decreasing by the previous inequality, it follows that $E(r) \leq E(0)$ for all $r \geq 0$. Note that equation (4.5) implies

$$
\Phi\left(\left|u^{\prime}(r)\right|\right)=\Phi\left(h^{-1}\left(\left|r^{-\alpha} \int_{0}^{r} \lambda t^{\gamma} f(u(t)) d t\right|\right)\right)
$$

which in turn gives

$$
\Phi\left(\left|u^{\prime}(r)\right|\right) \leq \Phi\left(h^{-1}\left(\frac{\lambda C_{\delta, d} r^{\gamma-\alpha+1}}{\gamma+1}\right)\right), r \in[0, \delta),
$$

where $C_{\delta, d}=\max _{r \in[0, \delta]}|f(u(r))|$. We choose $\delta>0$ small and apply Lemmas 5.1 and 5.2 to conclude from (4.11) that

$$
\Phi\left(\left|u^{\prime}(r)\right|\right) \leq\left(\frac{\lambda C_{\delta, d}}{\gamma+1}\right)^{\frac{\gamma_{2}}{\gamma_{1}-1}} r^{\frac{\gamma_{2}}{\gamma_{1}-1}(\gamma-\alpha+1)}, \forall r \in[0, \delta) .
$$

We apply condition $\Delta_{2}$ (see inequality (5.1) in the Appendix) in the definition of $E$ to infer that

$$
E(r) \leq\left(\gamma_{2}-1\right) r^{\alpha-\gamma} \Phi\left(\left|u^{\prime}(r)\right|\right)+\lambda F(u(r)), r>0 .
$$

Thus, (4.12) and (4.13) lead to

$$
E(r) \leq C r^{(\alpha-\gamma)+\frac{\gamma_{2}}{\gamma_{1}-1}(\gamma-\alpha+1)}+\lambda F(u(r)), r \in[0, \delta) .
$$

Recalling that $\gamma_{2} \geq \gamma_{1}$, we have that

$$
(\alpha-\gamma)+\frac{\gamma_{2},}{\gamma_{1}-1}(\gamma-\alpha+1) \geq \frac{\gamma_{1}-\alpha+\gamma}{\gamma_{1}-1}>0 .
$$

Hence, from (4.14) that $\lim _{r \rightarrow 0} E(r) \leq \lambda F(d)$. On the other hand, by Lemma 5.6, we know that $H(t) \geq 0$ for all $t \geq 0$. Then, by definition of $E, E(r) \geq \lambda F(u(r))$ for all $r>0$. Gathering these information, we conclude that

$$
\lim _{r \rightarrow 0} E(r)=\lambda F(d)
$$


Therefore, as (4.10) is true,

$$
E(r) \leq E(0) \text { for } r \geq 0,
$$

which is equivalent to the desired inequality namely (4.1)

Proof of Lemma 4.2 We will at first study existence and uniqueness of local solutions of $\left[P_{\lambda, d}\right]$. Let $\epsilon>0$ and consider

$$
\left\{\begin{array}{c}
-\left(r^{\alpha} \phi\left(\left|u^{\prime}(r)\right|\right) u^{\prime}(r)\right)^{\prime}=\lambda r^{\gamma} f(u(r)), \quad 0<r<\epsilon, \\
u(0)=d, \quad u^{\prime}(0)=0
\end{array}\right.
$$

We shall need the following result whose proof is left to the Appendix.

Lemma 4.3. $\left(P_{\lambda, d, \epsilon}\right.$ has a unique solution $u(\cdot)=u(\cdot, d, \lambda, \epsilon) \in C^{2}([0, \epsilon))$ for small $\epsilon$.

Proof of Uniqueness in Lemma 4.2 Assume that $u, v$ are two $C^{1}([0, \infty))$ solutions. Let

$$
S_{0}=\{r \geq 0: u(t)=v(t), 0 \leq t \leq r\}
$$

We will show that

$$
S_{0} \neq \emptyset, S_{0} \text { is both open and closed in }[0, \infty) .
$$

Indeed, by Lemma 4.3 above, $[0, \epsilon) \subset S_{0}$ for $\epsilon>0$ small enough, which shows that $S_{0} \neq \emptyset$. Moreover, since $u, v$ are $C^{1}$ functions we infer that $S_{0}$ is closed. To finish we shall prove that $S_{0}$ is open. To achieve that let $\widehat{r} \in S_{0}$ with $\widehat{r}>0$. We distinguish between two cases.

Case 1. $u^{\prime}(\widehat{r})=v^{\prime}(\widehat{r})=0$

Assume $u(\widehat{r})=v(\widehat{r})=\widehat{d}$. If $\widehat{d}=0$ then, up to a translation in the domain, we are within the settings of Lemma 4.1. Therefore, using (4.1), observing that by hypothesis $\left(f_{1}\right)$ one has $F(u(r)) \geq 0$ for $r \geq \widehat{r}$, and noticing that $F(\widehat{d})=0$, we get

$$
H\left(\left|u^{\prime}(r)\right|\right) \leq \lambda r^{\gamma-\alpha}(F(\widehat{d})-F(u(r))) \leq 0 \text { for } r \geq \widehat{r},
$$

from where it follows that $u(r)=0$ for $r \geq \widehat{r}$, because by Lemma 5.6 in the Appendix

$$
H(t) \geq 0 \forall t \geq 0 \text { and } H(t)=0 \Leftrightarrow t=0 .
$$

The same argument works to prove that $v(r)=0$ for $r \geq \widehat{r}$. Consequently, $r \geq \widehat{r}, u(r)=v(r)=0$ and then, $S_{0}=[0, \infty)$ is open. On the other hand, if $\widehat{d}>0$, we define

$$
\begin{gathered}
\widehat{K}_{\rho}^{\epsilon}(\hat{d})=\left\{u \in C([\widehat{r}, \widehat{r}+\epsilon]): u(0)=\widehat{d},\|u-\widehat{d}\|_{\infty} \leq \rho\right\}, \\
\widehat{T}(u(r))=\widehat{d}-\int_{\widehat{r}}^{r} h^{-1}\left(t^{-\alpha} \int_{\widehat{r}}^{t} \lambda \tau^{\gamma} f(u(\tau)) d \tau\right) d t, \forall r \in[0, \epsilon],
\end{gathered}
$$

where $\epsilon, \rho$ are positive and $\epsilon$ is small. The same proofs of (5.8) and (5.9) can be do here, and then the Banach Fixed Point Theorem guarantees a unique fixed point for the operator $\widehat{T}$ when $\epsilon$ is small, therefore, $u(r)=v(r)$ in a small neighbourhood of $\widehat{r}$, which implies that $S_{0}$ is open.

Case 2. $u^{\prime}(\widehat{r})=v^{\prime}(\widehat{r}) \neq 0$. 
Note that there is a neighbourhood $V$ of $\widehat{r}$ such that $u^{\prime}(r), v^{\prime}(r) \neq 0$ for $r \in V$. So in $V$, we must conclude, as in (4.10) (here we use the same notation as in the proof of Lemma 4.1) that if $z$ denotes either $u$ or $v$ then

$$
\left(r^{\alpha-\gamma} H\left(\left|z^{\prime}(r)\right|\right)+\lambda F(z(r))\right)^{\prime}=-\left.\frac{\alpha+\gamma\left(\gamma_{1}-1\right)}{\gamma_{1}} r^{\alpha-\gamma-1} \phi\left(\left|z^{\prime}(r)\right|\right) z^{\prime}(r)\right|^{2} .
$$

Integrating from $\widehat{r}$ to $r$ and subtracting the corresponding equations for $z=u$ and $z=v$, we obtain (remember that $u(\widehat{r})=v(\widehat{r})$ and $u^{\prime}(\widehat{r})=v^{\prime}(\widehat{r})$ )

$$
\begin{aligned}
& r^{\alpha-\gamma}\left[H\left(\left|u^{\prime}(r)\right|\right)-H\left(\left|v^{\prime}(r)\right|\right)\right]+\lambda[F(u(r))-F(v(r))]= \\
& -\frac{\alpha+\gamma\left(\gamma_{1}-1\right)}{\gamma_{1}} \int_{\widehat{r}}^{r} t^{\alpha-\gamma-1}\left[\phi\left(\left|u^{\prime}(t)\right|\right)\left|u^{\prime}(t)\right|^{2}-\phi\left(\left|v^{\prime}(t)\right|\right)\left|v^{\prime}(t)\right|^{2}\right] d t .
\end{aligned}
$$

Next we consider three auxliary continuous functions, namely

$$
\begin{aligned}
& A_{1}(t)=\left\{\begin{array}{cc}
\frac{H\left(\left|u^{\prime}(t)\right|\right)-H\left(\left|v^{\prime}(t)\right|\right)}{u^{\prime}(t)-v^{\prime}(t)}, & u^{\prime}(t) \neq v^{\prime}(t) \\
\phi\left(\left|u^{\prime}(t)\right|\right) u^{\prime}(t), & u^{\prime}(t)=v^{\prime}(t),
\end{array}\right. \\
& A_{2}(t)=\left\{\begin{array}{cl}
\frac{h\left(\left|u^{\prime}(t)\right|\right)\left|u^{\prime}(t)\right|-h\left(\left|v^{\prime}(t)\right|\right)\left|v^{\prime}(t)\right|}{u^{\prime}(t)-v^{\prime}(t)}, & u^{\prime}(t) \neq v^{\prime}(t) \\
\frac{d}{d t}\left[h\left(\left|u^{\prime}(t)\right|\right)\left|u^{\prime}(t)\right|\right], & u^{\prime}(t)=v^{\prime}(t),
\end{array}\right. \\
& B(t)=\left\{\begin{array}{cc}
\lambda \frac{F(u(t))-F(v(t))}{u(t)-v(t)}, & u(t) \neq v(t) \\
\lambda f(u(t)), & u(t)=v(t) .
\end{array}\right.
\end{aligned}
$$

Let $w(r)=u(r)-v(r)$. From (4.16),

$$
r^{\alpha-\gamma} A_{1}(r) w^{\prime}(r)+B(r) w(r)=-\frac{\alpha+\gamma\left(\gamma_{1}-1\right)}{\gamma_{1}} \int_{\hat{r}}^{r} t^{\alpha-\gamma-1} A_{2}(t) w^{\prime}(t) d t .
$$

Once $u^{\prime}(\widehat{r}) \neq 0$, we have that in a neighbourhood of $\widehat{r}$, the function $1 / A_{1}$ is well defined and continuous, and so, equation (4.17) is the same as

$$
w^{\prime}(r)+\frac{B(r)}{A_{1}(r)} r^{\gamma-\alpha} w(r)=-\frac{\alpha+\gamma\left(\gamma_{1}-1\right)}{\gamma_{1}} \frac{r^{\gamma-\alpha}}{A_{1}(r)} \int_{\hat{r}}^{r} t^{\alpha-\gamma-1} A_{2}(t) w^{\prime}(t) d t .
$$

As $h$ is two times differentiable and $u^{\prime}(\widehat{r}) \neq 0, A_{2}$ is continuously differentiable in a neighborhood of $\widehat{r}$, therefore, from (4.18) and integration by parts we obtain

$$
\begin{array}{r}
w^{\prime}(r)+\frac{B(r)}{A_{1}(r)} r^{\gamma-\alpha} w(r)=\frac{\alpha+\gamma\left(\gamma_{1}-1\right)}{\gamma_{1}} \frac{r^{\gamma-\alpha}}{A_{1}(r)} r^{\alpha-\gamma-1} A_{2}(r) w(r)+ \\
-\frac{\alpha+\gamma\left(\gamma_{1}-1\right)}{\gamma_{1}} \frac{r^{\gamma-\alpha}}{A_{1}(r)} \int_{\widehat{r}}^{r}\left[t^{\alpha-\gamma-1} A_{2}(t)\right]^{\prime} w(t) d t,
\end{array}
$$


hence

$$
\begin{aligned}
w^{\prime}(r)+ & {\left[\frac{B(r)}{A_{1}(r)} r^{\gamma-\alpha}-\frac{\alpha+\gamma\left(\gamma_{1}-1\right)}{\gamma_{1}} \frac{r^{\gamma-\alpha}}{A_{1}(r)} r^{\alpha-\gamma-1} A_{2}(r)\right] w(r)=} \\
& -\frac{\alpha+\gamma\left(\gamma_{1}-1\right)}{\gamma_{1}} \frac{r^{\gamma-\alpha}}{A_{1}(r)} \int_{\widehat{r}}^{r}\left[t^{\alpha-\gamma-1} A_{2}(t)\right]^{\prime} w(t) d t .
\end{aligned}
$$

We introduce the notation

$$
\begin{gathered}
D_{1}(r)=\frac{B(r)}{A_{1}(r)} r^{\gamma-\alpha}-\frac{\alpha+\gamma\left(\gamma_{1}-1\right)}{\gamma_{1}} \frac{r^{\gamma-\alpha}}{A_{1}(r)} r^{\alpha-\gamma-1} A_{2}(r), \\
D_{2}(r)=-\frac{\alpha+\gamma\left(\gamma_{1}-1\right)}{\gamma_{1}} \frac{r^{\gamma-\alpha}}{A_{1}(r)}, \\
D_{3}(r)=\left[t^{\alpha-\gamma-1} A_{2}(t)\right]^{\prime},
\end{gathered}
$$

which implies from (4.19) that

$$
w^{\prime}(r)+D_{1}(r) w(r)=D_{2}(r) \int_{\hat{r}}^{r} D_{3}(s) w(s) d s .
$$

We integrate equation (4.20) from $\widehat{r}$ to $r$, which combined with the fact that, $A_{1}, A_{2}, 1 / A_{1}, A_{2}^{\prime}, B$ are bounded functions (remember they are all continuous functions in a neighborhood of $\hat{r}$ ) to conclude that

$$
\begin{aligned}
|v(r)| & \leq \int_{\widehat{r}}^{r}\left|D_{1}(s)\right||v(s)| d s+\int_{\widehat{r}}^{r}\left|D_{2}(s)\right| \int_{\widehat{r}}^{s}\left|D_{3}(r)\right||v(t)| d t d s \\
& \leq C \int_{\widehat{r}}^{r}|v(s)| d s,
\end{aligned}
$$

where $C>0$ is a constant. By the Gronwall Inequality, $v=0$ in a neighborhood of $\hat{r}$. Therefore, $S_{0}$ is open and (4.15) is proved.

\section{Proof of Existence in Lemma 4.2. Let}

$$
S_{\infty}=\left\{r>0 \mid\left(\overline{P_{\lambda, d}}\right) \text { has a solution in }[0, r)\right\} \text { and } T_{\infty}=\sup S_{\infty}
$$

We will prove that

$$
T_{\infty}=\infty
$$

Assume, on the contrary, that $T_{\infty}<\infty$. First note that $S_{\infty}$ is a closed set. Indeed, let $r_{n} \rightarrow r$ with $r_{n} \in S_{\infty}$. If $r<r_{n}$ for some $n$ then $r \in S_{\infty}$ by force, so we can assume that $r_{n}<r$ and without loss of generality that $r_{n}<r_{n+1}$. If $u_{n}$ are the solutions associated with $r_{n}$, we define $u:[0, r) \rightarrow \mathbb{R}$ by $u(x):=u_{n}(x)$ of $x \in\left[0, r_{n}\right)$. Once (4.15) is satisfied, we conclude that $u$ is well defined and it is a solution of $\left(\overline{P_{\lambda, d}}\right)$, which implies that $r \in S_{\infty}$.

Since $S_{\infty}$ is closed, we have that $T_{\infty} \in S_{\infty}$. Let $u$ be the solution associated with $T_{\infty}$. We first observe that from (4.1), $\left|u^{\prime}\right|$ is bounded, which implies that $u$ can be continuously extended to $T_{\infty}$. Moreover, equation (4.5) guarantees that $u^{\prime}\left(T_{\infty}\right)$ is uniquely defined, so there are two cases to consider.:

Case 1. $u^{\prime}\left(T_{\infty}\right)=0$. 
If $u\left(T_{\infty}\right)=0$, consider the extension of $u$ namely $\widetilde{u}:[0, \infty) \rightarrow \mathbb{R}$ given by $\widetilde{u}(t)=0$ for $t \geq T_{\infty}$. Then $\widetilde{u}$ is a $C^{1}$ function and it is also a solution of $\left(\overline{P_{\lambda, d}}\right)$, which is an absurd. Otherwise, if $u\left(T_{\infty}\right)=d^{\infty}>0$, consider the operator $T$ defined by

$$
T(u(r))=d^{\infty}-\int_{T_{\infty}}^{r} h^{-1}\left(t^{-\alpha} \int_{T_{\infty}}^{t} \lambda \tau^{\gamma} f(u(\tau)) d \tau\right) d t
$$

Following the same lines as in the proof of either (5.8) or (4.15) Case 1, we have that $T$ has unique fixed point $v:\left[0, T_{\infty}+\epsilon\right]$, which is an absurd due to the definition of $T_{\infty}$.

Case 2. $u^{\prime}\left(T_{\infty}\right) \neq 0$.

Assume without loss of generality that $u^{\prime}\left(T_{\infty}\right)>0$. Then, by (4.5),

$$
u^{\prime}(r)=h^{-1}\left(r^{-\alpha} \int_{0}^{r} \lambda t^{\gamma} f(u(t)) d t\right)
$$

in a neighborhood of $T_{\infty}$. Hence, $u$ is $C^{2}$ in a neighborhood of $T_{\infty}$, which implies by $\left(P_{\lambda, d}\right)$ that

$$
u^{\prime \prime}(r)=-\left[\frac{d}{d t} h\left(u^{\prime}(r)\right)\right]^{-1}\left(\frac{\alpha}{r} \phi\left(u^{\prime}(r)\right) u^{\prime}(r)+\lambda r^{\gamma-\alpha} f(u(r))\right) .
$$

By the last equation and Peano's Theorem, $u$ can be extended to $\left[0, T_{\infty}+\delta\right)$, where $\delta>0$ and thus we reach an absurd, because such extension is also a solution to $\left(P_{\lambda, d}\right]$. This finishes the proof of Case 2. Therefore, (4.21) is proved and thus Claim 2 is also proved.

Proof of (4.3). Remember that

$$
r^{\alpha} \phi\left(\left|u^{\prime}(r)\right|\right) u^{\prime}(r)=-\int_{0}^{r} \lambda t \gamma f(u(t)) d t
$$

Assume that $d_{n} \rightarrow d_{0}$ and set $u_{n}(r)=u\left(r, d_{n}\right), u_{0}(r)=u\left(r, d_{0}\right)$. Inequality (4.5) implies that $\left|u_{n}^{\prime}(r)\right|$ is bounded for $r \in[0, T]$, therefore, by Áscoli-Arzéla Theorem, there is a subesequence, still denoted by $u_{n}$, such that $u_{n} \rightarrow v$ uniformly in $[0, T]$ for some $v \in C([0, T])$. Now we will prove that $v=u_{0}$.

First note that by Lebesgue's Theorem

$$
\int_{0}^{r} \lambda t \gamma f\left(u_{n}(t)\right) d t \rightarrow \int_{0}^{r} \lambda t \gamma f(v(t)) d t
$$

and by (4.22),

$$
r^{\alpha} \phi\left(\left|u_{n}^{\prime}(r)\right|\right) u_{n}^{\prime}(r) \rightarrow-\int_{0}^{r} \lambda t \gamma f(v(t)) d t, r \in[0, T]
$$

As a consequence,

$$
\left|u_{n}^{\prime}(r)\right| \rightarrow h^{-1}\left(r^{-\alpha}\left|\int_{0}^{r} \lambda t \gamma f(v(t)) d t\right|\right), r \in[0, T] .
$$

The combination of (4.22) and (4.23) implies that $u_{n}^{\prime}(r) \rightarrow w(r)$ for all $r \in[0, T]$ where $w$ is a continuous function. Hence, applying Lebesgue's Theorem we obtain

$$
u_{n}(r)-d_{n}=\int_{0}^{r} u_{n}^{\prime}(t) d t \rightarrow \int_{0}^{r} w(t) d r, r \in[0, T],
$$


which implies that $w(r)=v^{\prime}(r)$ and $v^{\prime}(0)=0$. Once

$$
\phi\left(\left|v^{\prime}(r)\right|\right) v^{\prime}(r)=-r^{-\alpha} \int_{0}^{r} \lambda t^{\gamma} f(v(t)) d t,
$$

is satisfied and since $v(0)=d_{0}$, it follows by the uniqueness of solutions given by theorem 4.2 that $v=u_{0}$, which concludes the proof of (4.3).

Proof of (4.4). Let $0<a \leq r \leq b<\infty$ and assume that $d_{n} \rightarrow d_{0}$. By (4.22),

$$
r^{\alpha}\left|\phi\left(\left|u_{n}^{\prime}(r)\right|\right) u_{n}^{\prime}(r)-\phi\left(\left|u_{0}^{\prime}(r)\right|\right) u_{0}^{\prime}(r)\right| \leq \int_{0}^{r} \lambda t^{\gamma}\left|f\left(u_{n}(t)\right)-f\left(u_{0}(t)\right)\right| d t .
$$

Since $\left(u_{n}\right)$ converges uniformly to $u_{0}$ in $[a, b]$, we conclude from the previous inequality that

$$
\left(\phi\left(\left|u_{n}^{\prime}(r)\right|\right) u_{n}^{\prime}(r)-\phi\left(\left|u_{0}^{\prime}(r)\right|\right) u_{0}^{\prime}(r)\right)\left(u_{n}^{\prime}(r)-u_{0}^{\prime}(r)\right) \rightarrow 0,
$$

uniformly in $[a, b]$. Now, we combine a generalized form of Simon's inequality, see Lemma [5.5 in the Appendix, with the last convergence to conclude that $u_{n}^{\prime} \rightarrow u_{0}^{\prime}$ uniformly in $[a, b]$. This finishes the proof of Lemma 4.2.

\subsection{Proof of Theorem 1.2 (Continued)}

Proof of (1.4). We will start by proving that there is $z_{1}=z_{1}(d)>0$ such that $u\left(z_{1}\right)=0$, $u^{\prime}\left(z_{1}\right)<0$ and

$$
u(r)>0, u^{\prime}(r)<0 \text { for } 0<r<z_{1} .
$$

Suppose, on the contrary, that $u(r)>0$ for all $r>0$. It follows from (4.22) and conditions $\left(f_{1}\right)$, $\left(f_{2}\right)$ that $u^{\prime}(r)<0$ and

$$
-u^{\prime}(r) \geq h^{-1}\left(\lambda \frac{r^{\gamma-\alpha+1}}{\gamma+1} f(u(r))\right), r>0 .
$$

Note that $u^{\prime}(r) \rightarrow 0$ if $r \rightarrow \infty$ because $u(r)>0$. Hence, the previous inequality implies that $u(r) \rightarrow 0$ if $r \rightarrow \infty$. Moreover, by Lemma 5.1 and the previous inequality, we also obtain

$$
-u^{\prime}(r) \geq \max \left\{\left(\frac{\lambda r^{\gamma-\alpha+1} f(u(r))}{(\gamma+1) h(1)}\right)^{\frac{1}{\gamma_{1}-1}},\left(\frac{\lambda r^{\gamma-\alpha+1} f(u(r))}{(\gamma+1) h(1)}\right)^{\frac{1}{\gamma_{2}-1}}\right\}, r>0,
$$

which implies

$$
-u^{\prime}(r) \min \left\{f(u(r))^{\frac{-1}{\gamma_{1}-1}}, f(u(r))^{\frac{-1}{\gamma_{1}-2}}\right\} \geq \min \left\{\left(\frac{\lambda r^{\gamma-\alpha+1}}{(\gamma+1) h(1)}\right)^{\frac{1}{\gamma_{1}-1}},\left(\frac{\lambda r^{\gamma-\alpha+1}}{(\gamma+1) h(1)}\right)^{\frac{1}{\gamma_{2}-1}}\right\}
$$

for each $r>0$. We integrate the last inequality from 0 to $r$ and apply the change of variables $t=u(s)$ to conclude that

$$
\int_{u(r)}^{d} \min \left\{f(t)^{\frac{-1}{\gamma_{1}-1}}, f(t)^{\frac{-1}{\gamma_{2}-1}}\right\} d t \geq \int_{0}^{r} \min \left\{\left(\frac{\lambda s^{\gamma-\alpha+1}}{(\gamma+1) h(1)}\right)^{\frac{1}{\gamma_{1}-1}},\left(\frac{\lambda s^{\gamma-\alpha+1}}{(\gamma+1) h(1)}\right)^{\frac{1}{\gamma_{2}-1}}\right\} d s .
$$

Hypothesis $(\gamma, \alpha)$ implies that the right hand side of (4.25) converges to infinity as $r \rightarrow \infty$. Therefore, (4.25) yields

$$
\liminf _{r \rightarrow \infty} \int_{u(r)}^{d} \min \left\{f(t)^{\frac{-1}{\gamma_{1}-1}}, f(t)^{\frac{-1}{\gamma_{2}-1}}\right\} d t=\infty
$$


which combined with $\left(f_{1}\right)$ and the fact that $u(r) \rightarrow 0$ if $r \rightarrow \infty$, implies a contradiction to $\left(f_{3}\right)$ and thus, (4.24) is true. To proceed, we will prove that there is $\Lambda>0$ such that

$$
z_{1}\left(d_{\infty}, \lambda\right) \geq R \text { if } 0<\lambda \leq \Lambda
$$

Indeed, by (4.22),

$$
-u^{\prime}(r) \leq h^{-1}\left(\frac{\lambda f\left(d_{\infty}\right) r^{\gamma-\alpha+1}}{\gamma+1}\right) \text { for } r \in\left[0, z_{1}\left(d_{\infty}, \lambda\right)\right]
$$

Integrating from 0 to $r \in\left[0, d_{\infty}\right]$ and making use of Lemma 5.1, we get that

$$
\begin{aligned}
-u(r)+d_{\infty} \leq \max \{ & \left(\gamma_{1}-1\right)\left(\frac{\lambda f\left(d_{\infty}\right)}{(\gamma+1) h(1)}\right)^{\frac{1}{\gamma_{1}-1}} \frac{r^{\frac{\gamma-\alpha+\gamma_{1}}{\gamma_{1}-1}}}{\gamma-\alpha+\gamma_{1}}, \\
& \left.\left(\gamma_{2}-1\right)\left(\frac{\lambda f\left(d_{\infty}\right)}{(\gamma+1) h(1)}\right)^{\frac{1}{\gamma_{2}-1}} \frac{r^{\frac{\gamma-\alpha+\gamma_{2}}{\gamma_{2}-1}}}{\gamma-\alpha+\gamma_{2}}\right\} .
\end{aligned}
$$

Let $\nu \in(0,1)$. Choose $r_{\infty}(\nu) \in\left(0, z_{1}\left(d_{\infty}, \lambda\right)\right)$ such that $u\left(r_{\infty}(\nu), d_{\infty}\right)=\nu d_{\infty}$. Set $r=r_{\infty}(\nu)$ in (4.27) and choose the maximum value in the right hand side of (4.27) which actually is

$$
\left(\gamma_{1}-1\right)\left(\frac{\lambda f\left(d_{\infty}\right)}{(\gamma+1) h(1)}\right)^{\frac{1}{\gamma_{1}-1}} \frac{r_{\infty}(\nu)^{\frac{\gamma-\alpha+\gamma_{1}}{\gamma_{1}-1}}}{\gamma-\alpha+\gamma_{1}}
$$

Take $R>0$ and choose $\Lambda_{\nu}>0$ satisfying

$$
1-\nu=\left[\left(\frac{\lambda f\left(d_{\infty}\right)}{(\gamma+1) h(1)}\right)^{\frac{1}{\gamma_{1}-1}} \frac{\gamma_{1}-1}{\gamma-\alpha+\gamma_{1}}\right]^{-1} \frac{\Lambda_{\nu}^{\frac{1}{\gamma_{1}-1}} R^{\frac{\gamma-\alpha+\gamma_{1}}{\gamma_{1}-1}}}{d_{\infty}} .
$$

We infer from (4.27) and (4.28) that

$$
\left[\left(\frac{\lambda f\left(d_{\infty}\right)}{(\gamma+1) h(1)}\right)^{\frac{1}{\gamma_{1}-1}} \frac{\gamma_{1}-1}{\gamma-\alpha+\gamma_{1}}\right]^{-1} \Lambda_{\nu}^{\frac{1}{\gamma_{1}-1}} R^{\frac{\gamma-\alpha+\gamma_{1}}{\gamma_{1}-1}} \leq\left(\gamma_{1}-1\right)\left(\frac{\lambda f\left(d_{\infty}\right)}{(\gamma+1) h(1)}\right)^{\frac{1}{\gamma_{1}-1}} \frac{r_{\infty}(\nu)^{\frac{\gamma-\alpha+\gamma_{1}}{\gamma_{1}-1}}}{\gamma-\alpha+\gamma_{1}}
$$

which implies that

$$
\Lambda_{\nu}^{\frac{1}{\gamma_{1}-1}} R^{\frac{\gamma-\alpha+\gamma_{1}}{\gamma_{1}-1}} \leq \lambda^{\frac{1}{\gamma_{1}-1}} r_{\infty}(\nu)^{\frac{\gamma-\alpha+\gamma_{1}}{\gamma_{1}-1}}
$$

Hence,

$$
R \leq r_{\infty}(\nu) \leq z_{1}\left(d_{\infty}, \lambda\right) \text { if } 0<\lambda \leq \Lambda_{\nu} .
$$

To finish the proof of (4.26), first note that the maximum of two continuous functions is a continuous function. Therefore, (4.27) combined with (4.28) gives

$$
\Lambda_{\nu}^{\frac{1}{\eta-1}} \stackrel{\nu \rightarrow 0}{\longrightarrow}\left(\frac{\lambda f\left(d_{\infty}\right)}{(\gamma+1) h(1)}\right)^{\frac{1}{\eta-1}} \frac{\eta-1}{\gamma-\alpha+\eta} \frac{d_{\infty}}{R^{\frac{\gamma-\alpha+\eta}{\eta-1}}}
$$

where either $\eta=\gamma_{1}$ or $\eta=\gamma_{2}$ depending on whether the maximum in (4.27) is assumed at $\gamma_{1}$ or $\gamma_{2}$. Note also that $r_{\nu}\left(d_{\infty}\right)$ is continuous on $\nu$ and $r_{\nu}\left(d_{\infty}\right) \rightarrow z_{1}\left(d_{\infty}, \lambda\right)$ as $\nu \rightarrow 0$, therefore, we conclude from (4.29) that

$$
R \leq z_{1}\left(d_{\infty}, \lambda\right) \text { if } 0<\lambda \leq \Lambda
$$


where

$$
\Lambda:=\frac{\lambda f\left(d_{\infty}\right)}{(\gamma+1) h(1)}\left(\frac{\eta-1}{\gamma-\alpha+\eta}\right)^{\eta-1} \frac{d_{\infty}^{\eta-1}}{R^{\gamma-\alpha+\eta}}
$$

Now we will show that there is $z_{2}=z_{2}(d)>z_{1}$ such that $u\left(z_{2}\right)=0, u^{\prime}\left(z_{2}\right)>0$ and

$$
u(r)<0, z_{1}<r<z_{2} .
$$

In fact, since $u^{\prime}\left(z_{1}\right)<0$ then, $u^{\prime}(r)<0$ in a neighborhood of $z_{1}$. We start by proving that there is $m_{1}>z_{1}$ such that $u^{\prime}\left(m_{1}\right)=0$. Thus, suppose by contradiction that it is not true, i.e. $u^{\prime}(r)<0$ for all $r>z_{1}$. We have by (4.2) that

$$
\int_{0}^{u(r)} f(t) d t \leq F(d), r \geq 0
$$

If there is some sequence $r_{n} \rightarrow \infty$ such that $u\left(r_{n}\right) \rightarrow-\infty$ then, by the previous inequality we infer that

$$
\int_{-\infty}^{0} f(s) d s=\lim _{n} \int_{u\left(r_{n}\right)}^{0} f(s) d s \geq-F(d),
$$

which is impossible, because $\left(f_{1}\right),\left(f_{2}\right)$ imply that $\int_{-\infty}^{0} f(s) d s=-\infty$. Hence, there is $C>0$ such that

$$
u(r) \geq-C, u^{\prime}(r)<0, \forall r \geq z_{1},
$$

and consequently $u(r) \rightarrow L$ as $r \rightarrow \infty$ for some $L<0$. Now, by (4.1),

$$
\frac{\Phi\left(\left|u^{\prime}(r)\right|\right)}{r^{\gamma-\alpha+1}} \rightarrow 0 \text { as } r \rightarrow \infty,
$$

which implies by using the inequality $\Phi(s) \geq c s^{2} \phi(s)$ that

$$
\frac{\phi\left(\left|u^{\prime}(r)\right|\right)}{r^{\gamma-\alpha+1}} \rightarrow 0
$$

On one hand (4.22) and the previous limits imply that

$$
\frac{1}{r^{\gamma+1}} \int_{0}^{r} t^{\gamma} f(u(t)) d t \rightarrow 0
$$

and on the other side, $\left(f_{1}\right)$ and L'Hospital rule imply that

$$
\lim _{r \rightarrow \infty} \frac{1}{r^{\gamma+1}} \int_{0}^{r} t^{\gamma} f(u(t)) d t=\lim _{r \rightarrow \infty} \frac{r^{\gamma} f(u(r))}{(\gamma+1) r^{\gamma}}=\frac{f(L)}{\gamma+1}<0,
$$

which is an absurd. Therefore, $u^{\prime}\left(m_{1}\right)=0$ for some $z_{1}<m_{1}$, so that

$$
u(r)<0 \text { for } z_{1}<r<m_{1} \text { and } u^{\prime}(r)<0 \text { for } z_{1} \leq r<m_{1} .
$$

Now, taking $r>m_{1}, r$ close to $m_{1}$ we have

$$
\int_{m_{1}}^{r} t^{\gamma} f(u(t))<0
$$

which implies by (4.22) that

$$
u(r)<0, u^{\prime}(r)>0 \text { for all } r>m_{1}, r \text { close to } m_{1} .
$$


Assume by contradiction that $u(r)<0$ for $r>m_{1}$, so that $u^{\prime}(r)>0$. Since by $\left(f_{2}\right)$

$$
-r^{\alpha} \phi\left(\left|u^{\prime}(r)\right|\right) u^{\prime}(r)=\lambda \int_{m_{1}}^{r} t^{\gamma} f(u(t)) d t \leq \frac{\lambda f(u(r))}{\gamma+1}\left(r^{\gamma+1}-m_{1}^{\gamma+1}\right),
$$

we get by taking $r>\bar{r}=2^{\frac{1}{\gamma+1}} m_{1}$ above, that $r^{\gamma+1}-m_{1}^{\gamma+1}>\frac{r^{\gamma+1}}{2}$ and so

$$
-r^{\alpha} \phi\left(\left|u^{\prime}(r)\right|\right) u^{\prime}(r) \leq \frac{\lambda f(u(r))}{2(\gamma+1)} r^{\gamma+1}
$$

which, combined with Lemma 5.1 gives

$$
u^{\prime}(r) \geq \min \left\{\left(\frac{-\lambda f(u(r))}{2(\gamma+1)} r^{\gamma-\alpha+1}\right)^{\frac{1}{\gamma_{1}-1}},\left(\frac{-\lambda f(u(r))}{2(\gamma+1)} r^{\gamma-\alpha+1}\right)^{\frac{1}{\gamma_{2}-1}}\right\}, r>\bar{r} .
$$

Integrating in (4.31) from $\bar{r}$ to $r$, we have

$$
\int_{\bar{r}}^{r} u^{\prime}(t) \max \left\{(-f(u(t)))^{\frac{-1}{\gamma_{1}-1}},(-f(u(t)))^{\frac{-1}{\gamma_{2}-1}}\right\} d t \geq \int_{\bar{r}}^{r} \min \left\{\left(\frac{t^{\gamma-\alpha+1}}{2(\gamma+1)}\right)^{\frac{1}{\gamma_{1}-1}},\left(\frac{t^{\gamma-\alpha+1}}{2(\gamma+1)}\right)^{\frac{1}{\gamma_{2}-1}}\right\} d t
$$

for $r>\bar{r}$. Making the change of variables $y=u(t)$,

$$
\int_{u(\bar{r})}^{u(r)} \max \left\{(-f(t))^{\frac{-1}{\gamma_{1}-1}},(-f(t))^{\frac{-1}{\gamma_{2}-1}}\right\} d t \geq \int_{\bar{r}}^{r} \min \left\{\left(\frac{t^{\gamma-\alpha+1}}{2(\gamma+1)}\right)^{\frac{1}{\gamma_{1}-1}},\left(\frac{t^{\gamma-\alpha+1}}{2(\gamma+1)}\right)^{\frac{1}{\gamma_{2}-1}}\right\} d t .
$$

Once $u(r)<0$ and $u^{\prime}(r)>0$ for $r>\bar{r}$ it follows that $u^{\prime}(r) \rightarrow 0$ as $r \rightarrow \infty$. Hence, inequality (4.31) implies that $u(r) \rightarrow 0$ as $r \rightarrow \infty$. Moreover, the right hand side of (4.32) converges to $\infty$ due to hypothesis $(\gamma, \alpha)$. Therefore

$$
\liminf \int_{u(\bar{r})}^{u(r)} \max \left\{(-f(t))^{\frac{-1}{\gamma_{1}-1}},(-f(t))^{\frac{-1}{\gamma_{2}-1}}\right\}=\infty,
$$

which contradicts $\left(f_{3}\right)$, so (4.30) is proved. Now we will prove that there is $z_{3}=z_{3}(d)>z_{2}$ such that $u\left(z_{3}\right)=0, u^{\prime}\left(z_{3}\right)<0$ and

$$
u(r)>0 \text { for all } r \in\left(z_{2}, z_{3}\right) .
$$

Indeed, since by (4.30), $u^{\prime}\left(z_{2}\right)>0$, so that

$$
u^{\prime}(r)>0 \text { for all } r>z_{2}, r \text { close to } z_{2} .
$$

We claim that there is $m_{2}>z_{2}$ such that $u^{\prime}\left(m_{2}\right)=0$. In fact, othewise, $u^{\prime}(r)>0$, for all $r>z_{2}$, which gives that $u(r)>0$ for $r>z_{2}$. By (4.2),

$$
\int_{0}^{u(r)} f(t) d t \leq \int_{0}^{d} f(t) d t
$$

so that $u(r) \leq d$ for $r \geq z_{2}$. Hence, there is $L \in(0, d]$ such that

$$
u(r) \rightarrow L \text { and } u(r) \leq L, r \geq z_{2} .
$$


As in the proof of (4.30),

$$
\frac{1}{r^{\gamma+1}} \int_{0}^{r} t^{\gamma} f(u(t)) d t \rightarrow 0
$$

and

$$
\lim _{r \rightarrow \infty} \frac{1}{r^{\gamma+1}} \int_{0}^{r} t^{\gamma} f(u(t)) d t=\lim _{r \rightarrow \infty} \frac{r^{\gamma} f(u(r))}{(\gamma+1) r^{\gamma}}=\frac{f(L)}{\gamma+1}<0,
$$

which is an absurd. As a consequence, there is $m_{2}>z_{2}$ such that $u^{\prime}\left(m_{2}\right)=0$ and $u^{\prime}(r)>0$, $z_{2} \leq r<m_{2}$, proving the claim. Assume again, by contradiction, that $u(r)>0$ for all $r>m_{2}$ so that $u^{\prime}(r)<0$ also for all $r>m_{2}$. We have (similar to the proof of (4.30)

$$
-r^{\alpha} \phi\left(\left|u^{\prime}(r)\right|\right) u^{\prime}(r)=\lambda \int_{m_{2}}^{r} t^{\gamma} f(u(t)) d t \geq \frac{\lambda f(u(r))}{\gamma+1}\left(r^{\gamma+1}-m_{2}^{\gamma+1}\right) .
$$

Setting $\bar{r}=2^{\frac{1}{\gamma+1}} m_{2}$ and taking $r>\bar{r}$,

$$
-r^{\alpha} \phi\left(\left|u^{\prime}(r)\right|\right) u^{\prime}(r) \geq \frac{\lambda f(u(r))}{2(\gamma+1)} r^{\gamma+1}
$$

which combined with (5.1) gives

$$
-u^{\prime}(r) \geq \min \left\{\left(\frac{\lambda f(u(r))}{2(\gamma+1)} r^{\gamma-\alpha+1}\right)^{\frac{1}{\gamma_{1}-1}},\left(\frac{\lambda f(u(r))}{2(\gamma+1)} r^{\gamma-\alpha+1}\right)^{\frac{1}{\gamma_{2}-1}}\right\}, r>\bar{r} .
$$

Integrating (4.34) from $\bar{r}$ to $r$ and making the change of variables $u(t)=s$, we get

$$
\int_{u(\bar{r})}^{u(r)}-\max \left\{f(t)^{\frac{-1}{\gamma_{1}-1}}, f(t)^{\frac{-1}{\gamma_{2}-1}}\right\} d t \geq \int_{\bar{r}}^{r} \min \left\{\left(\frac{t^{\gamma-\alpha+1}}{2(\gamma+1)}\right)^{\frac{1}{\gamma_{1}-1}},\left(\frac{t^{\gamma-\alpha+1}}{2(\gamma+1)}\right)^{\frac{1}{\gamma_{2}-1}}\right\} d t .
$$

Taking lim inf in both sides, we arrive at a contradiction with $\left(f_{3}\right)$ and so (4.33) is true. To finish the proof of (4.4) we argue as in (4.30) and (4.33) to get zeroes $z_{4}, z_{5}$ and inductively, a sequence with the properties asserted in (1.4).

Proof of (1.5). We start by proving that $z_{1}(d) \rightarrow 0$ when $d \rightarrow 0$. By (4.22) and (4.24) we obtain

$$
-u^{\prime}(r)=h^{-1}\left(r^{-\alpha} \int_{0}^{r} \lambda t^{\gamma} f(u(t)) d t\right), r \in\left[0, z_{1}\right] .
$$

Now we apply $\left(f_{2}\right)$ and Lemma 5.1 to conclude that

$$
-u^{\prime}(r) \geq \min \left\{\left(\lambda \frac{r^{\gamma-\alpha+1} f(u(r))}{\gamma+1}\right)^{\frac{1}{\gamma_{1}-1}},\left(\lambda \frac{r^{\gamma-\alpha+1} f(u(r))}{\gamma+1}\right)^{\frac{1}{\gamma_{2}-1}}\right\}, r \in\left[0, z_{1}\right],
$$

which implies that

$$
-u^{\prime}(r) \max \left\{f(u(r))^{\frac{-1}{\gamma_{1}-1}}, f(u(r))^{\frac{-1}{\gamma_{2}-1}}\right\} \geq \min \left\{\left(\lambda \frac{r^{\gamma-\alpha+1}}{\gamma+1}\right)^{\frac{1}{\gamma_{1}-1}},\left(\lambda \frac{r^{\gamma-\alpha+1}}{\gamma+1}\right)^{\frac{1}{\gamma_{2}-1}}\right\}, r \in\left[0, z_{1}\right] .
$$

Integrating from 0 to $r$ and making the change of variables $y=u(t)$ we get to

$$
\int_{u(r)}^{d} \max \left\{f(t)^{\frac{-1}{\gamma_{1}-1}}, f(t)^{\frac{-1}{\gamma_{2}-1}}\right\} d t \geq \int_{0}^{r} \min \left\{\left(\lambda \frac{t^{\gamma-\alpha+1}}{\gamma+1}\right)^{\frac{1}{\gamma_{1}-1}},\left(\lambda \frac{t^{\gamma-\alpha+1}}{\gamma+1}\right)^{\frac{1}{\gamma_{2}-1}}\right\} d t .
$$


Taking $r=z_{1}(d)$ in the previous inequality and making use of $(\gamma, \alpha)$ and $\left(f_{3}\right)$, we conclude that $z_{1}(d) \rightarrow 0$ as $d \rightarrow 0$. Now, letting $\ell \geq 1$, we assume that $u(r)>0$ in $\left(z_{\ell}(d), z_{\ell+1}(d)\right)$, so that by the notations of (4.30) and (4.33) we have $u^{\prime}(r)>0$ in $\left(z_{\ell}(d), m_{\ell}(d)\right)$ and $u^{\prime}(r)<0$ in $\left(m_{l}(d), z_{\ell+1}(d)\right)$ (the case $u(r)<0$ in $\left(z_{\ell}(d), z_{\ell+1}(d)\right)$ is handled similarly). Now, using $\left(f_{2}\right)$ in (4.22), taking $m_{\ell}(d) \leq r \leq z_{\ell+1}(d)$ and then applying lemma 5.1, we obtain successively

$$
\begin{gathered}
r^{\alpha} h\left(-u^{\prime}(r)\right) \geq \lambda f(u(r)) \frac{r^{\gamma+1}-m_{\ell}(d)^{\gamma+1}}{\gamma+1}, \\
-u^{\prime}(r) \max \left\{f(u(r))^{\frac{-1}{\gamma_{1}-1}}, f(u(r))^{\frac{-1}{\gamma_{2}-1}}\right\} \geq \min \left\{\left(\lambda \frac{r^{\gamma+1}-m_{\ell}(d)^{\gamma+1}}{(\gamma+1) r^{\alpha}}\right)^{\frac{1}{\gamma_{1}-1}},\left(\lambda \frac{r^{\gamma+1}-m_{\ell}(d)^{\gamma+1}}{(\gamma+1) r^{\alpha}}\right)^{\frac{1}{\gamma_{2}-1}}\right\} .
\end{gathered}
$$

Note that $r^{\gamma-\alpha} \geq m_{\ell}(d)^{\gamma-\alpha}$ since $\gamma \geq \alpha$, therefore

$$
r^{\gamma-\alpha+1}-r^{-\alpha} m_{\ell}(d)^{\gamma+1} \geq m_{\ell}(d)^{\gamma-\alpha}\left(r-m_{\ell}(d)\right),
$$

which gives

$$
\begin{aligned}
& -u^{\prime}(r) \max \left\{f(u(r))^{\frac{-1}{\gamma_{1}-1}}, f(u(r))^{\frac{-1}{\gamma_{2}-1}}\right\} \geq \\
& \min \left\{\left[\frac{\lambda m_{\ell}(d)^{\gamma-\alpha}}{(\gamma+1)}\left(r-m_{\ell}(d)\right)\right]^{\frac{1}{\gamma_{1}-1}},\left[\frac{\lambda m_{\ell}(d)^{\gamma-\alpha}}{(\gamma+1)}\left(r-m_{\ell}(d)\right)\right]^{\frac{1}{\gamma_{2}-1}}\right\} .
\end{aligned}
$$

Integrating from $m_{\ell}(d)$ to $z_{\ell+1}(d)$, making the change of variables $y=u(t)$, we find that

$$
\begin{aligned}
& \int_{0}^{u\left(m_{\ell}(d)\right)} \max \left\{f(t)^{\frac{-1}{\gamma_{1}-1}}, f(t)^{\frac{-1}{\gamma_{2}-1}}\right\} d t \geq \\
& \int_{m_{\ell}(d)}^{z \ell+1}(d) \min \left\{\left[\frac{\lambda m_{\ell}(d)^{\gamma-\alpha}}{(\gamma+1)}\left(r-m_{\ell}(d)\right)\right]^{\frac{1}{\gamma_{1}-1}},\left[\frac{\lambda m_{\ell}(d)^{\gamma-\alpha}}{(\gamma+1)}\left(r-m_{\ell}(d)\right)\right]^{\frac{1}{\gamma_{2}-1}}\right\} d t .
\end{aligned}
$$

Assume now $z_{\ell}(d)<r<m_{\ell}(d)$. Then by a similar argument, this time, integrating from $z_{\ell}(d)$ to $m_{\ell}(d)$ we deduce that

$$
\begin{aligned}
& \int_{0}^{u\left(m_{\ell}(d)\right)} \max \left\{f(t)^{\frac{-1}{\gamma_{1}-1}}, f(t)^{\frac{-1}{\gamma_{2}-1}}\right\} d t \geq \\
& \int_{z_{\ell}(d)}^{m_{\ell}(d)} \min \left\{\left[\frac{\lambda m_{\ell}(d)^{\gamma-\alpha}}{(\gamma+1)}\left(m_{\ell}(d)-r\right)\right]^{\frac{1}{\gamma_{1}-1}},\left[\frac{\lambda m_{\ell}(d)^{\gamma-\alpha}}{(\gamma+1)}\left(m_{\ell}(d)-r\right)\right]^{\frac{1}{\gamma_{2}-1}}\right\} d t .
\end{aligned}
$$

Now, since $u\left(m_{\ell}(d)\right) \leq d$ we have by $\left(f_{3}\right)$ that the left hand side of (4.36) and (4.37) converge to zero, and therefore, $\lim _{d \rightarrow 0} z_{\ell}(d)=\lim _{d \rightarrow 0} z_{\ell+1}(d)$ for each $\ell \geq 1$. Once $z_{1}(d) \rightarrow 0$ as $d \rightarrow 0$, we see that $z_{\ell}(d) \rightarrow 0$ as $d \rightarrow 0$.

We pass to the proof that $z_{\ell}(d) \rightarrow z_{\ell}\left(d_{0}\right)$ if $d \rightarrow d_{0}$. Let us first show that $z_{1}(d) \rightarrow z_{1}\left(d_{0}\right)$ as $d \rightarrow d_{0}$. Indeed, let $d_{n} \rightarrow d_{0}, u_{n}(\cdot)=u\left(\cdot, d_{n}\right)$ and $u_{0}(\cdot)=u\left(\cdot, d_{0}\right)$ so that we have from (4.3) that $u_{n} \rightarrow u$ uniformly in compact subsets of $(0, \infty)$. For each $\epsilon>0$ small we find

$$
u_{0}(r)>0,0 \leq r \leq z_{1}\left(d_{0}\right)-\epsilon \text { and } u_{0}\left(z_{1}\left(d_{0}\right)+\epsilon\right)<0
$$


so that

$$
u_{n}(r)>0,0 \leq r \leq z_{1}\left(d_{0}\right)-\epsilon \text { and } u_{n}\left(z_{1}\left(d_{0}\right)+\epsilon\right)<0
$$

for sufficiently large $n$. As a consequence, $z_{1}\left(d_{0}\right)-\epsilon<z_{1}\left(d_{n}\right)<z_{1}\left(d_{0}\right)+\epsilon$, showing that $z_{1}\left(d_{n}\right) \rightarrow z_{1}\left(d_{0}\right)$. Now, assume by induction that $z_{\ell}\left(d_{n}\right) \rightarrow z_{\ell}\left(d_{0}\right)$ for some $\ell>1$. We will show that $z_{\ell+1}\left(d_{n}\right) \rightarrow z_{\ell+1}\left(d_{0}\right)$. For that matter, we assume $u_{0}(t)<0$ for $z_{\ell}\left(d_{0}\right)<t<z_{\ell+1}\left(d_{0}\right)$ (the other case is handled similarly). Taking $\epsilon>0$ small, we find that $u_{n}(t)<0$ for $z_{\ell}\left(d_{0}\right)+\epsilon \leq t \leq z_{\ell+1}\left(d_{0}\right)-\epsilon$ and $u_{n}\left(z_{\ell+1}\left(d_{0}\right)+\epsilon\right)>0$, showing that $z_{\ell+1}\left(d_{0}\right)-\epsilon<z_{\ell+1}\left(d_{n}\right)<z_{\ell+1}\left(d_{0}\right)+\epsilon$. Consequently, $z_{\ell+1}\left(d_{n}\right) \rightarrow z_{\ell+1}\left(d_{0}\right)$ as $d \rightarrow d_{0}$, which finishes the proof of (1.5).

\section{Proof of (1.6).}

Let $d \in\left(0, d_{0}\right)$. It suffices to show that $z_{\ell+2}(d)>R$ whenever $d$ is close enough to $d_{0}$. We assume that $u\left(r, d_{0}\right)<0$ for $r \in\left(z_{\ell}\left(d_{0}\right), z_{\ell+1}\left(d_{0}\right)\right)$ (the other case is handled similarly).

Notice that as $z_{\ell}\left(d_{0}\right)$ is increasing an there is only $\ell$ zeroes in $(0, R)$, we must show that $z_{\ell+1}\left(d_{0}\right) \geq R$ and $z_{\ell+2}\left(d_{0}\right)>R$. However, as $z_{\ell+2}(d) \rightarrow z_{\ell+2}\left(d_{0}\right)$ for $d \rightarrow d_{0}$, we have $z_{\ell+2}(d)>R$ whenever $d$ is close enough to $d_{0}$. This completes the proof of Theorem 1.2 .

\section{Appendix}

Remark 5.1. (On the radially symetric form of $(\Phi)$ ) Let u be a weak solution of $(\Phi)$, radially symmetric in the sense that $u(x)=u(|x|)=u(r)$. Let $r \in(0, R)$ and pick $\epsilon>0$ small such that $0<r<r+\epsilon<R$.

Consider the radially symmetric cut-off function $v_{r, \epsilon}(x)=v_{r, \epsilon}(r)$, where

$$
v_{r, \epsilon}(t):=\left\{\begin{array}{l}
1 \text { if } 0 \leq t \leq r \\
\text { linear if } r \leq t \leq r+\epsilon \\
0 \text { if } r+\epsilon \leq t \leq R
\end{array}\right.
$$

and notice that $v_{r, \epsilon} \in W_{0}^{1, \Phi}(B) \mid \cap \operatorname{Lip}(\bar{B})$. By replacing $v$ with $v_{r, \epsilon}$ in (2.2), we get to

$$
\frac{-1}{\epsilon} \int_{B(0, r+\epsilon) \backslash B(0, r)} \phi\left(\left|u^{\prime}(|x|)\right|\right) u^{\prime}(|x|) d x=\lambda \int_{B(0, r+\epsilon)} f(u(|x|)) v_{r, \epsilon}(|x|) d x .
$$

Making the change of variables $x=r \omega$ with $r>0$ and $\omega \in \partial B(0,1)$ and letting $\epsilon \rightarrow 0$ we infer that

$$
\phi\left(\left|u^{\prime}(r)\right|\right) u^{\prime}(r) r^{N-1}=\lambda \int_{0}^{r} f(u(r)) r^{N-1} d r
$$

which gives

$$
\left(r^{N-1} \phi\left(\left|u^{\prime}(r)\right|\right) u^{\prime}(r)\right)^{\prime}=\lambda r^{N-1} f(u(r)) .
$$

So the radially symetric form of $(\Phi)$ is

$$
\left\{\begin{array}{c}
-\left(r^{N-1} \phi\left(\left|u^{\prime}(r)\right|\right) u^{\prime}(r)\right)^{\prime}=\lambda r^{N-1} f(u(r), 0<r<R \\
u^{\prime}(0)=u(R)=0 .
\end{array}\right.
$$

Lemma 5.1. Assume that $\phi$ satisfies $\left(\phi_{1}\right)-\left(\phi_{3}\right)$. Then

$$
h(1) \min \left\{h^{-1}(s)^{\gamma_{1}-1}, h^{-1}(s)^{\gamma_{2}-1}\right\} \leq s \leq h(1) \max \left\{h^{-1}(s)^{\gamma_{1}-1}, h^{-1}(s)^{\gamma_{2}-1}\right\}, s>0 .
$$


Proof. Condition $\left(\phi_{3}\right)$ implies that

$$
\left(\gamma_{1}-1\right) \frac{d}{d t} \ln t \leq \frac{d}{d t} \ln h(t) \leq\left(\gamma_{2}-1\right) \frac{d}{d t} \ln t, \forall t>0 .
$$

Let $t \leq 1$. Integrating the previous inequality from $t$ to 1 , we get

$$
h(1) t^{\gamma_{1}-1} \leq h(t) \leq h(1) t^{\gamma_{2}-1}, t \leq 1 .
$$

Let $t \geq 1$. Iintegrating the previous inequality from 1 to $t$, we get

$$
h(1) t^{\gamma_{2}-1} \leq h(t) \leq h(1) t^{\gamma_{1}-1}, \forall t \geq 1 .
$$

Therefore

$$
h(1) \min \left\{t^{\gamma_{1}-1}, t^{\gamma_{2}-1}\right\} \leq h(t) \leq h(1) \max \left\{t^{\gamma_{1}-1}, t^{\gamma_{2}-1}\right\}, \forall t>0 .
$$

Letting $t=h^{-1}(s)$, the lemma is proved.

Lemma 5.2. Assume $\phi$ satisfies $\left(\phi_{1}\right)-\left(\phi_{3}\right)$. Then

$$
\Phi(1) \min \left\{t^{\gamma_{1}}, t^{\gamma_{2}}\right\} \leq \Phi(t) \leq \Phi(1) \max \left\{t^{\gamma_{1}}, t^{\gamma_{2}}\right\}, t>0 .
$$

Proof. From $\left(\phi_{3}\right)$,

$$
\gamma_{1} t \phi(t) \leq t h^{\prime}(t)+t \phi(t) \leq \gamma_{2} t \phi(t), \forall t>0,
$$

which implies, after integration from 0 to $t$ that,

$$
\gamma_{1} \leq \frac{t \Phi^{\prime}(t)}{\Phi(t)} \leq \gamma_{2}, t>0
$$

The previous inequality is called condition $\Delta_{2}$. To finish the proof, we proceed as in the proof of lemma 5.1 to conclude the desired inequality.

Lemma 5.3. Assume that $\phi$ satisfies $\left(\phi_{1}\right)-\left(\phi_{3}\right)$. Then

$$
\left[h^{-1}\right]^{\prime}(t) \leq \frac{t^{\frac{-\gamma_{2}+2}{\gamma_{2}-1}}}{h(1)^{\gamma_{2}\left(\gamma_{1}-1\right)}}, t \leq 1 .
$$

Proof. Remember that

$$
\left[h^{-1}\right]^{\prime}(t)=\frac{1}{h^{\prime}\left(h^{-1}(t)\right)}, t>0 .
$$

From the proofs of Lemmas 5.1 and 5.2 ,

$$
h(1)\left(\gamma_{1}-1\right) \min \left\{t^{\gamma_{1}-2}, t^{\gamma_{2}-2}\right\} \leq h^{\prime}(t) \leq h(1)\left(\gamma_{2}-1\right) \max \left\{t^{\gamma_{1}-2}, t^{\gamma_{2}-2}\right\} \text { for } t>0 .
$$

Gathering (5.2) and (5.3), we see that

$$
\left[h^{-1}\right]^{\prime}(t) \leq \frac{\left[h^{-1}(t)\right]^{-\gamma_{2}+2}}{h(1)\left(\gamma_{1}-1\right)}, t \leq 1
$$

Now we use Lemma 5.1 to obtain

$$
\left[h^{-1}\right]^{\prime}(t) \leq \frac{t^{\frac{-\gamma_{2}+2}{\gamma_{2}-1}}}{h(1)^{\gamma_{2}}\left(\gamma_{1}-1\right)}, t \leq 1 .
$$


Lemma 5.4. Assume that $\phi:(0, \infty) \rightarrow(0, \infty)$ is a differentiable function satisfying $\left(\phi_{3}\right)$. Then, there is a positive constant $\Gamma_{1}$ such that

$$
\sum_{i, j=1}^{N} \frac{\partial a_{j}}{\partial \eta_{i}}(\eta) \xi_{i} \xi_{j} \geq \Gamma_{1} \phi(|\eta|)|\xi|^{2},
$$

where $a_{j}(\eta)=\phi(|\eta|) \eta_{j}, \eta \in \mathbb{R}^{N} \backslash\{0\}$ and $\xi \in \mathbb{R}^{N}$.

Proof. Indeed, by $\left(\phi_{3}\right)$,

$$
\left(\gamma_{1}-2\right) \phi(t) \leq t \phi^{\prime}(t) \leq\left(\gamma_{2}-2\right) \phi(t) .
$$

Suppose first that $\gamma_{1}<2$. Note that

$$
\sum_{i, j=1}^{N} \frac{\partial a_{j}}{\partial \eta_{i}}(\eta) \xi_{i} \xi_{j}=\phi(|\eta|)|\xi|^{2}+\frac{\phi^{\prime}(|\eta|)|\langle\eta, \xi\rangle|^{2}}{|\eta|}
$$

If $\phi^{\prime}(|\eta|)<0$, then $\phi^{\prime}(|\eta|)|\langle\eta, \xi\rangle|^{2} \geq \phi^{\prime}(|\eta|)|\eta|^{2}|\xi|^{2}$. From (15.5) and (5.6),

$$
\sum_{i, j=1}^{N} \frac{\partial a_{j}}{\partial \eta_{i}}(\eta) \xi_{i} \xi_{j} \geq\left(\gamma_{1}-1\right) \phi(|\eta|)|\xi|^{2} .
$$

If $\phi^{\prime}(|\eta|) \geq 0$, then take $\Gamma_{1}=1$.

If $\gamma_{1} \geq 2$, then (5.5) is satisfied with $\Gamma_{1}=1$, as can readily be seen from (5.6) and noting that $\phi^{\prime}(t) \geq 0$ in this case.

We now prove a Simon type inequality.

Lemma 5.5. Assume that $\phi:(0, \infty) \rightarrow(0, \infty)$ is a differentiable function satisfying $\left(\phi_{1}\right)-\left(\phi_{3}\right)$. Then

$$
\left\langle\phi(|\eta|) \eta-\phi\left(\left|\eta^{\prime}\right|\right) \eta^{\prime}, \eta-\eta^{\prime}\right\rangle \geq \min \left\{4,4 \Gamma_{1}\right\} \frac{\left|\eta-\eta^{\prime}\right|}{1+|\eta|+\left|\eta^{\prime}\right|} \Phi\left(\frac{\left|\eta-\eta^{\prime}\right|}{4}\right),
$$

where $\Gamma_{1}$ was given in lem.a 5.4. $\eta, \eta^{\prime} \in \mathbb{R}^{N}$ and $\langle\cdot, \cdot\rangle$ denotes inner product.

Proof. If $\eta, \eta^{\prime}=0$ then (5.7) is obviously satisfied. If only one of them is 0 , let's say, $\eta^{\prime}=0$, then

$$
\phi(|\eta|)|\eta|^{2} \geq \Phi(|\eta|) \geq 4 \Phi\left(\frac{|\eta|}{4}\right)
$$

where in the last inequalities we have used the properties of an $\mathrm{N}$-function (note that an $\mathrm{N}$-function is convex). So (5.7) is satisfied. If $\eta, \eta^{\prime} \neq 0$, assume without loss of generality that $|\eta| \leq\left|\eta^{\prime}\right|$. Then, an application of Cauchy-Schwartz inequality implies that

$$
\frac{\left|\eta-\eta^{\prime}\right|}{4} \leq\left|t \eta+(1-t) \eta^{\prime}\right| \leq 1+|\eta|+\left|\eta^{\prime}\right|, t \in[0,1 / 4] .
$$


We conclude from the last inequality, (5.6) and the properties of an N-function that

$$
\begin{aligned}
\left\langle\phi(|\eta|) \eta-\phi\left(\left|\eta^{\prime}\right|\right) \eta^{\prime}, \eta-\eta^{\prime}\right\rangle & =\sum_{i=1}^{N} \int_{0}^{1} \frac{d}{d t}\left[a_{j}\left(t \eta+(1-t) \eta^{\prime}\right)\right]\left(\eta_{j}-\eta_{j}^{\prime}\right) d t \\
& =\int_{0}^{1} \sum_{i, j=1}^{N} \frac{\partial a_{j}}{\partial \eta_{i}}\left[t \eta+(1-t) \eta^{\prime}\right]\left(\eta_{i}-\eta_{i}^{\prime}\right)\left(\eta_{j}-\eta_{j}^{\prime}\right) d t \\
& \geq \Gamma_{1} \int_{0}^{1} \phi\left(\left|t \eta+(1-t) \eta^{\prime}\right|\right)\left|\eta-\eta^{\prime}\right|^{2} d t \\
& \geq \Gamma_{1} \int_{0}^{1 / 4} \phi\left(\left|t \eta+(1-t) \eta^{\prime}\right|\right)\left|\eta-\eta^{\prime}\right|^{2} d t \\
& =\Gamma_{1} \int_{0}^{1 / 4} \phi\left(\left|t \eta+(1-t) \eta^{\prime}\right|\right)\left|\eta-\eta^{\prime}\right|^{2} \frac{\left|t \eta+(1-t) \eta^{\prime}\right|}{\left|t \eta+(1-t) \eta^{\prime}\right|} d t \\
& \geq 4 \Gamma_{1} \frac{\left|\eta-\eta^{\prime}\right|}{1+|\eta|+\left|\eta^{\prime}\right|} \phi\left(\frac{\left|\eta-\eta^{\prime}\right|}{4}\right)\left(\frac{\left|\eta-\eta^{\prime}\right|}{4}\right)^{2} \\
& \geq 4 \Gamma_{1} \frac{\left|\eta-\eta^{\prime}\right|}{1+|\eta|+\left|\eta^{\prime}\right|} \Phi\left(\frac{\left|\eta-\eta^{\prime}\right|}{4}\right) .
\end{aligned}
$$

Lemma 5.6. Assume $\phi$ satisfies $\left(\phi_{1}\right)$ - $\left(\phi_{3}\right)$. Then, the function $H(t)=t \Phi^{\prime}(t)-\Phi(t)$ is strictly increasing and satisfies

$$
\begin{aligned}
& \left(\gamma_{1}-1\right) \Phi(t) \leq H(t) \leq\left(\gamma_{2}-1\right) \Phi(t), t \geq 0, \\
& \frac{\gamma_{1}-1}{\gamma_{1}} t \Phi^{\prime}(t) \leq H(t) \leq \frac{\gamma_{2}-1}{\gamma_{2}} t \Phi^{\prime}(t), \quad t \geq 0 .
\end{aligned}
$$

Proof. Indeed, as $\left(\phi_{3}\right)$ is satisfied, we have that

$$
t \Phi^{\prime}(t)-r \Phi^{\prime}(r)>(t-r) \Phi^{\prime}(t)>\int_{r}^{t} \tau \phi(\tau) d \tau, t>r \geq 0
$$

which implies that $H$ is strictly incresing. On the other hand, condition (5.1) implies the desired inequalities.

Proof of Lemma 4.3 Indeed, take $\rho \in(0, d)$ and set

$$
K_{\rho}^{\epsilon}(d)=\left\{u \in C([0, \epsilon]) \mid u(0)=d,\|u-d\|_{\infty} \leq \rho\right\} .
$$

Take $\epsilon>0$ small. If $u \in K_{\rho}^{\epsilon}(d)$, then by continuity, $u(r)>0, r \in[0, \epsilon]$. Hence, for small $\epsilon$, a solution of $P_{\lambda, d, \epsilon}$ satisfies $u^{\prime}(r) \leq 0$ for $r \in[0, \epsilon]$ (this was showed in the proof of proposition (4.1)) and

$$
u(r)=d-\int_{0}^{r} h^{-1}\left(t^{-\alpha} \int_{0}^{t} \lambda \tau^{\gamma} f(u(\tau)) d \tau\right) d t, \forall r \in[0, \epsilon] .
$$

We infer that the solutions of $\left(\overline{P_{\lambda, d, \epsilon}}\right)$, for small $\epsilon$, are fixed points of the operator

$$
T(u(r))=d-\int_{0}^{r} h^{-1}\left(t^{-\alpha} \int_{0}^{t} \lambda \tau^{\gamma} f(u(\tau)) d \tau\right) d t, \forall r \in[0, \epsilon]
$$


Now we will verify that there exist $\epsilon, \rho>0$ and $k \in(0,1)$ such that

$$
T\left(K_{\rho}^{\epsilon}(d)\right) \subset K_{\rho}^{\epsilon}(d)
$$

and

$$
\|T u-T v\|_{\infty} \leq k\|u-v\|_{\infty}
$$

Therefore, by the Banach Fixed Point Theorem, $T$ has a unique fixed point, which in turn will be a $C^{2}([0, \epsilon])$ solution of $\left(\overline{\left.P_{\lambda, d, \epsilon}\right)}\right.$. With respect to (5.8), let $\rho \in(0, d / 2]$, which implies that $u(r) \in[d / 2,2 d]$ for $u \in K_{\rho}^{\epsilon}(d)$. Therefore, for $u \in K_{\rho}^{\epsilon}(d)$ we have that

$$
h^{-1}\left(r^{-\alpha} \int_{0}^{s} \lambda t^{\gamma} f(u(t)) d t\right) \leq h^{-1}\left(\frac{\lambda\|f\|_{\infty, d} s^{\gamma-\alpha+1}}{\gamma+1}\right), s \in[0, \epsilon]
$$

where $\|f\|_{\infty, d}=\max _{s \in[d / 2,2 d]} f(s)$. For small $\epsilon$, we can apply lemma 5.1 in the Appendix to conclude from the previous inequality that

$$
\begin{aligned}
|T(u(r))-T(u(0))| & =\int_{0}^{r} h^{-1}\left(r^{-\alpha} \int_{0}^{s} \lambda t^{\gamma} f(u(t)) d t\right) d s \\
& \leq \int_{0}^{r} h^{-1}\left(\frac{\lambda\|f\|_{\infty, d} s^{\gamma-\alpha+1}}{\gamma+1}\right) d s \\
& \leq \int_{0}^{r} h(1)\left(\frac{\lambda\|f\|_{\infty, d} s^{\gamma-\alpha+1}}{\gamma+1}\right)^{\frac{1}{\gamma_{1}-1}} d s \\
& =h(1)\left(\frac{\lambda\|f\|_{\infty, d}}{\gamma+1}\right)^{\frac{1}{\gamma_{1}-1}} r^{\frac{\gamma-\alpha+\gamma_{1}}{\gamma_{1}-1}}, r \in[0, \epsilon] .
\end{aligned}
$$

As $\gamma \geq \alpha$, we obtain from the last inequality that there is $\epsilon>0$ such that $T u \in C([0, \epsilon])$ and $|T(u(r))-d| \leq \rho$ for $r \in[0, \epsilon]$, which finishes the proof of (5.8). Now we pass to the proof of (5.9). We first prove it by assuming that $f \in C(\mathbb{R}) \cap C^{1}(\mathbb{R} \backslash\{0\})$. Fix $\rho$ as in (5.8) and take $u, v \in K_{\rho}^{\epsilon}(d)$. By the Mean Value Theorem, there is $h \in(0,1)$ such that

$$
\begin{array}{r}
T(v(r))-T(u(r))=\int_{0}^{r}\left[h^{-1}\left(s^{-\alpha} \int_{0}^{s} \lambda t^{\gamma} f(u(t)) d t\right)-h^{-1}\left(s^{-\alpha} \int_{0}^{s} \lambda t^{\gamma} f(v(t)) d t\right)\right] d s= \\
\int_{0}^{r}\left[\left(h^{-1}\right)^{\prime}\left(s^{-\alpha} \int_{0}^{s} \lambda t^{\gamma} f(h u(t)+(1-h) v(t)) d t\right)\left(s^{-\alpha} \int_{0}^{s} \lambda t^{\gamma} f^{\prime}(h u(t)+(1-h) v(t))(u(t)-v(t)) d t\right)\right] d s .
\end{array}
$$

Choose $\epsilon$ small in such a way that the number $s^{-\alpha} \int_{0}^{s} \lambda t^{\gamma} f(h u(t)+(1-h) v(t)) d t$ for $s \in[0, \epsilon]$ is small. Therefore, Lemma 5.3 and the last equality implies that for $1<\gamma_{2} \leq 2$ (note that in this case, the function $t \mapsto t^{\frac{-\gamma_{2}+2}{\gamma_{2}-1}}$ is decreasing) 


$$
\begin{gathered}
|T(v(r))-T(u(r))| \leq \\
\int_{0}^{r}\left[c\left(s^{-\alpha} \int_{0}^{s} \lambda t^{\gamma}|f(h u(t)+(1-h) v(t))| d t\right)^{\frac{-\gamma_{2}+2}{\gamma_{2}-1}}\left(s^{-\alpha} \int_{0}^{s} \lambda t^{\gamma}\left|f^{\prime}(h u(t)+(1-h) v(t)) \| u(t)-v(t)\right| d t\right)\right] d s \leq \\
\int_{0}^{r}\left[c\left(s^{-\alpha} \int_{0}^{s} \lambda\|f\|_{\infty, d^{\prime}} t^{\gamma} d t\right)^{\frac{-\gamma_{2}+2}{\gamma_{2}-1}}\left(s^{-\alpha} \int_{0}^{s} \lambda\left\|f^{\prime}\right\|_{\infty, d} t^{\gamma} d t\right)\|u-v\|_{\infty}\right] d s= \\
\int_{0}^{r}\left[c\left(\lambda\|f\|_{\infty, d^{\prime}} \frac{s^{-\alpha+\gamma+1}}{\gamma+1}\right)^{\frac{-\gamma_{2}+2}{\gamma_{2}-1}}\left(\lambda\left\|f^{\prime}\right\|_{\infty, d} \frac{s^{-\alpha+\gamma+1}}{\gamma+1} d t\right)\|u-v\|_{\infty}\right] d s= \\
c\left(\frac{\lambda\|f\|_{\infty, d^{\prime}}}{\gamma+1}\right)^{\frac{-\gamma_{2}+2}{\gamma_{2}-1}} \frac{\lambda\left\|f^{\prime}\right\|_{\infty, d}}{\gamma+1} r \frac{-\alpha+\gamma+\gamma_{2}}{\gamma_{2}-1}\|u-v\|_{\infty},
\end{gathered}
$$

where $\|f\|_{\infty, d^{\prime}}=\min _{s \in[d / 2,2 d]}|f(s)|$ and $\left\|f^{\prime}\right\|_{\infty, d}=\max _{s \in[d / 2,2 d]}\left|f^{\prime}(s)\right|$. If on the other hand, we have that $\gamma_{2} \geq 2$, i.e., $t \mapsto t^{\frac{-\gamma_{2}+2}{\gamma_{2}-1}}$ is increasing then, we must conclude that

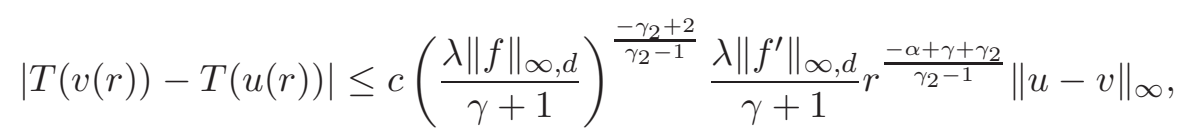

where $\|f\|_{\infty, d}=\max _{\in[d / 2, d]} f(s)$. In both cases, hypothesis $\gamma(\gamma, \alpha)$ implies the existence of $\epsilon$ such that (5.9) is true in the case $f \in C(\mathbb{R}) \cap C^{1}(\mathbb{R} \backslash\{0\})$.

\section{References}

[1] J. V. Goncalves \& C. A. Santos, Positive solutions of some quasilinear singular second order equations. Journal of the Australian Mathematical Society 76 (2001) 125-140.

[2] J. V. Goncalves \& C. A. Santos, Classical solutions of singular Monge-Ampére equations in a ball, Journal of Mathematical Analysis and Applications, 305 (2005) 240-252.

[3] J. V. Goncalves \& A. L. Melo, Multiple sign changing solutions in a class of quasilinear equations, Differential Integral Equations, 15 (2002) 147-165.

[4] N. Fukagai \& K. Narukawa, On the existence of multiple positive solutions of quasilinear elliptic eigenvalue problems, Annali di Matematica 186 (2007) 539-564.

[5] Y. Cheng, On the existence of radial solutions of a nonlinear elliptic equation on the unit ball, Nonlinear Anal. 24 (1995) 287 - 307.

[6] J. Iaia, Radial solutions to a p-Laplacian Dirichlet problem, Applicable Anal. 58 (1995) 335 350 .

[7] A. Castro \& A. Kurepa, Infinitely many radially symmetric solutions to a superlinear Dirichlet problem in a ball, Proc. Amer. Math. Soc. 101 (1987) 57 -64.

[8] Ph. Clément, D.G. de Figueiredo \& E. Mitidieri, Quasilinear elliptic equations with critical exponents, Top. Meth. Nonl. Anal. 7 (1996), 133-170. 
[9] A. Castro, J. Cossio \& J. M. Neuberger, A sign-changing solution for a superlinear Dirichlet problem, Rocky Mountain J. Math. 27 (4) (1997), 1041-1053.

[10] M. Mihailescu \& V. Radulescu; Nonhomogeneous Neumann problems in Orlicz-Sobolev spaces, C .R. Acad. Sci. Paris, Ser. I 346 (2008), 401-406.

[11] M. Mihailescu \& V. Radulescu;Existence and multiplicity of solutions for a quasilinear nonhomogeneous problems: An Orlicz-Sobolev space setting, J. Math. Anal. Appl. 330 (2007), 416-432.

[12] W. M. Ni \& J. Serrin, Nonexistence theorems for singular solutions of quasilinear partial differential equations, Comm. Pure Appl. Math. 39 (1986) 379 - 399.

[13] J. Simon, Regularité de la solution d'une equation non lineaire dans $\mathbf{R}^{\mathbf{N}}$, Springer Lecture Notes in Math. \# 665 (Ph. Benilan Editor) (1978), 203 - 227.

[14] W. Strauss, Existence of solitary waves in higher dimensions, Comm. Math. Phys. 55 (1977) $149-162$.

[15] P. Tolksdorff, On quasilinear boundary value problems in domains with corners, Nonlinear Anal. 5 (1981) 721-735.

[16] R. Saxton \& D. Wei, Radial solutions to a nonlinear p-harmonic Dirichlet problem, Applicable Anal. 51 (1993) $59-80$.

Claudianor O. Alves
Universidade Federal de Campina Grande
Unidade Acadêmica de Matemática
$58109-970$ Campina Grande, PB - Brazil
email: coalves@yahoo.com.br
Jose V. A. Goncalves
Universidade Federal de Goiás
Instituto de Matemática e Estatística
74001-970 Goiânia, GO - Brazil
email: goncalves.jva@gmail.com
Kaye O. Silva
Instituto Federal Goiano
Núcleo de Estatística, Matemática e Matemática Aplicada
$74085-010$ Urutaí, GO - Brazil
email: kayeoliveira@hotmail.com

